\title{
XBP1s promotes the development of lung adenocarcinoma via the p-JNK MAPK pathway
}

\author{
HONGXIA JIANG $^{1,2}$, QIANQIAN JIANG ${ }^{1,2}$, YUANZHOU HE $\mathrm{HE}^{1,2}$, \\ XIAOCHEN LI ${ }^{1,2}$, YONGJIAN XU ${ }^{1,2}$ and XIANSHENG LIU ${ }^{1,2}$ \\ ${ }^{1}$ Department of Pulmonary and Critical Care Medicine, Tongji Hospital, Tongji Medical College, \\ Huazhong University of Science and Technology; ${ }^{2}$ Key Laboratory of Pulmonary Diseases, \\ National Ministry of Health of The People's Republic of China, Wuhan, Hubei 430030, P.R. China
}

Received October 14, 2021; Accepted December 16, 2021

DOI: $10.3892 / \mathrm{ijmm} .2022 .5089$

\begin{abstract}
Spliced X-box binding protein 1 (XBP1s) has been reported to participate in the pathogenesis of numerous types of cancer; however, whether XBP1s plays a role in lung cancer remains to be elucidated. In the present study, bioinformatics analysis was performed to determine the mRNA expression level of XBP1 in lung cancer and adjacent normal tissues. Gene Ontology terms, pathway enrichment and Pearson's correlation analysis were performed to investigate the possible mechanism involved. Western blot and reverse transcription-quantitative PCR were performed to quantify the protein and mRNA expression level of target proteins, respectively. Small interfering RNA or overexpression plasmid were used to knockdown or overexpress the expression level of XBP1s. EdU staining, colony formation, Cell Counting Kit-8, Transwell and wound healing assays, and flow cytometry were performed to detect the proliferation, colony forming ability, cell viability, migration and invasion ability, and the apoptosis rate. The results showed that the mRNA and protein expression level of XBP1 was higher in tumor tissues compared with that in adjacent normal tissues using data from the TIMER2.0,
\end{abstract}

Correspondence to: Dr Xiansheng Liu, Department of Pulmonary and Critical Care Medicine, Tongji Hospital, Tongji Medical College, Huazhong University of Science and Technology, 1095 Jiefang Avenue, Wuhan, Hubei 430030, P.R. China

E-mail: doctorliu69@126.com

Abbreviations: XBP1s, spliced X-box binding protein 1; ERS, endoplasmic reticulum stress; IRE1, inositol-requiring enzyme 1; PERK, double-stranded RNA-activated protein kinase-like ER kinase; ATF6, activating transcription factor 6; RT-qPCR, reverse transcription-quantitative PCR; HIF1 $\alpha$, hypoxia inducible factor $\alpha$; TNM, Tumor Node Metastasis; LUAD, lung adenocarcinoma; NSCLC, non-small cell lung cancer; KEGG, Kyoto Encyclopedia of Genes and Genomes; HBE, human bronchial epithelial; TCGA, The Cancer Genome Atlas; FDR, False Discovery Rate

Key words: XBP1s, LUAD, hypoxia, p-JNK, MAPK, A549, bioinformatic analysis
ONCOMINE and UALCAN online databases. In addition, the mRNA expression level of XBP1 was also associated with clinical features, including age, smoking habit, individual cancer stage and nodal metastasis status. In the in vitro experiments, the mRNA and protein expression level of XBP1s was increased in the A549 cell line compared with that in the human bronchial epithelial (HBE), H1299, PC9 and H460 cell lines. Hypoxia further increased the protein expression level of XBP1s in the A549 cell line. Knockdown of XBP1s expression in the A549 cell line resulted in decreased proliferation, colony formation, cell viability, migration and invasion, and increased apoptosis. By contrast, overexpressing XBP1s in the HBE cell line led to the opposite results. To investigate the mechanism involved, proteins associated with XBP1 were analyzed using the LinkedOmics database. Pathway enrichment revealed the MAPK pathway to be the possible XBP1 downstream target. Furthermore, Pearson's correlation and western blot analyses verified that phosphorylated (p)-JNK rather than p-ERK or p-p38 was the downstream effector of XBP1s. Phosphorylation of JNK was decreased when XBP1s expression was knocked down in the A549 cell line under normoxic and hypoxic conditions. Inhibiting p-JNK with SP600125 reversed the increased prosurvival effects caused by XBP1s overexpression. The results from the present study suggest that XBP1s/p-JNK function as a prosurvival factors in the A549 cell line and could be a potential target for the treatment of lung adenocarcinoma.

\section{Introduction}

Lung cancer was the most commonly diagnosed cancer (11.6\% of total cancer cases) and the leading cause of cancer-related death (18.4\% of total cancer-related deaths) worldwide according to the global cancer statistics from 2018 (1). Among all lung cancer cases, at least $80 \%$ are non-small cell lung cancer (NSCLC) (2) and among these, lung adenocarcinoma (LUAD) accounts for $50 \%$ of all the cases (3). The high prevalence rate of LUAD requires further investigation to elucidate the mechanisms that drive its pathogenesis and to determine potential targeted therapies.

The endoplasmic reticulum is a complex organelle that functions to orchestrate protein folding, $\mathrm{Ca} 2^{+}$storage, and lipid and carbohydrate metabolism. When cells are exposed 
to intracellular or extracellular stimuli that cause unfolded or misfolded proteins to accumulate in the endoplasmic reticulum lumen, a condition known as endoplasmic reticulum stress (ERS) is initiated to restore homeostasis. There are three endoplasmic reticulum membrane-embedded sensors which transduce signals during ERS, including inositol-requiring enzyme 1 (IRE1), double-stranded RNA-activated protein kinas-like ER kinase (PERK) and activating transcription factor 6 (ATF6) (4). In rapidly growing solid tumors, low levels of oxygen and glucose often trigger ERS and lead to the activation of spliced X-box binding protein (XBP1s) (5).

XBP1s is a key transcription factor during ERS. Upon ERS, IRE1 $\alpha$ splices a 26-base intron from the XBP1 mRNA, transforming it from its unspliced form (XBPlu) to its spliced form (XBP1s) (6). XBP1s was reported to participate in the development of numerous types of cancer (7). IRE1 $\alpha$-XBP1 was reported to promote prostate cancer by activating the c-MYC pathway (8) and control T cell function in ovarian cancer by regulating mitochondrial activity (9). XBP1 was also reported to promote triple-negative breast cancer by regulating the hypoxia inducible factor $\alpha$ (HIF1 $\alpha$ ) pathway (10). Several studies have revealed that XBP1s plays a significant role in tumorigenesis and cancer progression; however, to the best of our knowledge, research on the role of XBP1s in LUAD is limited. A recent study revealed that XBP1s was overexpressed in NSCLC tissues and associated with Tumor Node Metastasis (TNM) stages, lymph node metastasis and poor prognosis (11). However, the mechanisms involved requires further investigation.

There are three main branches of MAPKs, including JNK, ERK and p38 MAPK. These enzymes regulate various cellular activities, including proliferation, differentiation, apoptosis, survival, inflammation and innate immunity. These proteins contribute to the pathology of diverse human diseases, including cancer (12). Numerous studies have proven MAPK to be associated with the initiation and progression of LUAD (13-20). However, whether there is an association between XBP1s and MAPK in LUAD has not yet been investigated.

The present study was designed to investigate whether and how XBP1s participates in the development of LUAD and whether MAPK was involved in this process.

\section{Materials and methods}

Datasets. Both mRNA and protein expression of XBP1 were analyzed using bioinformatics analysis. The mRNA expression level of XBP1 in various types of cancer was analyzed using the ONCOMINE (https://www.oncomine. org/resource/login.html) (21) and the TIMER2.0 databases (https://cistrome.shinyapps.io/timer) (22). The thresholds in the ONCOMINE database were set as follows: $\mathrm{P}<0.05$, fold change of all and gene rank of all. The protein expression level of XBP1 in patients with LUAD and normal tissue from the Clinical Proteomic Tumor Analysis Consortium, and the association between XBP1 and the clinical characteristics was analyzed using the UALCAN database (https:/ualcan.path. uab.edu) (23). The proteins associated with XBP1, and Gene Ontology (biological processes) analysis, and pathway enrichment were analyzed (false discovery rate $<0.01$ ) using the LinkedOmics database (https://www.linkedomics.org/login. php) (24). RNA sequencing data in patients with LUAD from
TCGA database (https://www.cancer.gov/about-nci/organization/ccg/research/structural-genomics/tcga) were analyzed. The pathways involved in LUAD were analyzed using the Kyoto Encyclopedia of Genes and Genomes (KEGG) database (https://kegg.jp) (25). The association between XBP1 and the MAPK pathway was analyzed using the LinkedOmics and GEPIA databases (https://gepia.cancer-pku.cn) (26).

Cell culture. The human LUAD cell lines, A549, H1299 and PC9, and the large cell lung cancer cell line, H460 were purchased from American Type Culture Collection. Human bronchial epithelial (HBE) cell line was purchased from NTCC Preservation Center. RPMI-1640 (Nanjing KeyGen Biotech Co., Ltd.), supplemented with 10\% FBS (Gibco; Thermo Fisher Scientific, Inc.) was used to culture the A549, H1299 and H460 cell lines. DMEM/High Glucose (HyClone; Cytiva), supplemented with 10\% FBS was used to culture the HBE and PC9 cell lines. The cells were routinely cultured at $37^{\circ} \mathrm{C}$ in a humidified incubator (Thermo Fisher Scientific, Inc.) with $21 \% \mathrm{O}_{2}, 5 \% \mathrm{CO}_{2}$ and $74 \% \mathrm{~N}_{2}$. For the hypoxic culture environment, the A549 cell line was cultured at $37^{\circ} \mathrm{C}$ in a humidified incubator (Thermo Fisher Scientific, Inc.) for $0,12,24,36,48$ and $60 \mathrm{~h}$ with $2 \% \mathrm{O}_{2}, 5 \% \mathrm{CO}_{2}$ and $93 \% \mathrm{~N}_{2}$. SP600125 (cat. no. HY-12041; MedChemExpress) was used to inhibit the JNK MAPK pathway in rescue experiments.

Small interfering (si)RNA and plasmid transfection. siRNAs targeting XBP1s were designed by Guangzhou RiboBio Co. Ltd. The following sequences were used: siNC (non-targeting), 5'-TTCTCCGAACGTGTCACGTdTdT-3'; siXBP1s-1, 5'-GCA AGTGGTAGATTTAGAA-3'; siXBP1s-2, 5'-GATCGAAAG AAGGCTCGAA-3'; and siXBP1s-3, 5'-TGAGAACCAGGA GTTAAGA-3'. siRNA $(50 \mu \mathrm{M})$ was transfected into the A549 cell line seeded $\left(2.0 \times 10^{5}\right.$ cells/well) in the 6-well plate. The cells were incubated with transfection reagent in the incubator for $24 \mathrm{~h}$ at $37^{\circ} \mathrm{C}$ with $21 \% \mathrm{O}_{2}$. Then, the medium was replaced with fresh culture medium and prepared for subsequent experiments. siRNA transfection was conducted using Lipofectamine $^{\mathrm{TM}} 3000$ reagent (cat. no. 2241260; Invitrogen; Thermo Fisher Scientific, Inc.).

The A549 and HBE cell lines were transfected with either blank (no vector), liposome, siNC and siXBP1s-1/2/3 or control and XBP1s overexpression vector to knockdown the expression level of XBP1s or increase the expression level of $\mathrm{XBP} 1 \mathrm{~s}$, respectively.

The XBP1s overexpression plasmid was designed by Shanghai GeneChem Co., Ltd., using Pcdna3.1-flag plasmid. Plasmid transfection was conducted using Lipofectamine $^{\mathrm{TM}} 3000$ and P3000 ${ }^{\mathrm{TM}}$ reagent (cat. no. 2241260; Invitrogen; Thermo Fisher Scientific, Inc.), and the cells were transfected with $800 \mathrm{ng}$ control or XBP1s overexpression vector. Fresh culture medium was replaced $8 \mathrm{~h}$ following transfection and the cells were cultured for another $16 \mathrm{~h}$ at $37^{\circ} \mathrm{C}$, then used for subsequent experiments.

Western blot analysis. Protein from the cells was extracted with protein extraction buffer (RIPA, protease inhibitor cocktail, PMSF, phosphorylation protease inhibitor A and B; ratio, 100:2:1:1:1. Protein concentration was determined using a BCA protein Concentration kit (cat. no. 16F17B97; Boster Biological 
Technology). Protein (30 $\mu \mathrm{g})$ was loaded into each lane and separated using $10 \%$ SDS-PAGE, then transferred onto a PVDF membrane. The membrane was blocked with $5 \%$ skimmed milk for $1 \mathrm{~h}$ at room temperature, washed with TBS with $0.05 \%$ Tween-20 (TBST) and incubated with the primary antibodies overnight at $4^{\circ} \mathrm{C}$. The following primary antibodies were used: XBP1s (cat. no. 40435s; 1:1,000; Cell Signaling Technology, Inc.), phosphorylated (p)-c-JNK (cat. no. 4668; 1:1,000; Cell Signaling Technology, Inc.), JNK (cat. no. 9252; 1:1,000; Cell Signaling Technology, Inc.), p-ERK (cat. no. 4370; 1:1,000; Cell Signaling Technology, Inc.), ERK (cat. no. 4695; 1:1,000; Cell Signaling Technology, Inc.), p-p38 (cat. no. 4511; 1:1,000; Cell Signaling Technology, Inc.), p38 (cat. no. 8690; 1:1,000; Cell Signaling Technology, Inc.) and actin (cat. no. 66009-1-Ig; 1:4,000; ProteinTech Group, Inc.). Then, the membrane was washed with TBST, incubated with HRP-conjugated secondary antibodies (goat anti-rabbit, cat. no. AS1107; goat anti-mouse, cat. no. AS1106) (both 1:4,000 and purchased from Wuhan Aspen Biotechnology, Co., Ltd.) for $1 \mathrm{~h}$ at room temperature. The membranes were washed with TBST again and the protein bands were detected using a detection kit (cat. no. 201005-79; Advansta, Inc.). ImageJ software (v1.46r; National Institutes of Health) was used to semi-quantify protein expression.

Reverse transcription-quantitative PCR (RT-qPCR). Total RNA was extracted using TRIzol ${ }^{\circledR}$ (Invitrogen; Thermo Fisher Scientific, Inc.). Then, RT using PrimeScript RT Master Mix (cat. no. RR036A; Takara Biotechnology Co., Ltd.) was performed with $500 \mathrm{ng}$ RNA and a total volume of $10 \mu \mathrm{l}$. The following temperature conditions were used: $37^{\circ} \mathrm{C}$ for $15 \mathrm{~min}, 85^{\circ} \mathrm{C}$ for $5 \mathrm{sec}$ and held at $4^{\circ} \mathrm{C}$ until further experimentation. qPCR was performed using TB Green ${ }^{\circledR}$ Premix Ex Taq (cat. no. RR420A; Takara Biotechnology Co., Ltd.) and the following thermocycling conditions: Initial denaturation at $95^{\circ} \mathrm{C}$ for $30 \mathrm{sec}$, followed by 40 cycles at $95^{\circ} \mathrm{C}$ for $5 \mathrm{sec}$ and $60^{\circ} \mathrm{C}$ for $30 \mathrm{sec}$, and a final extension at $65-95^{\circ} \mathrm{C}$, in $0.5^{\circ} \mathrm{C}$ increments for $5 \mathrm{sec}$. Relative quantification analysis was performed using the $2^{-\Delta \Delta \mathrm{Cq}}$ method (27). The following primers were used to amplify the genes of interest: XBP1s, forward, 5'-GCTGAG TCCGCAGCAGG-3' and reverse, 5'-CTCTGGGGAAGG GCATTTGA-3'; actin forward, 5'-AGCGAGCATCCCCCA AAGTT-3' and reverse, 5'-GGGCACGAAGGCTCATCATT-3'.

EdU staining assay and cytotoxicity assay. The cells were seeded into 96-well plates, transfected with siXBP1s or siNC (A549 cells; 6,000 cells/well), or XBP1s overexpression vector or control vector (A549 and HBE cells) (both 8,000 cells/well). The cells were then cultured under $21 \% \mathrm{O}_{2}$ normoxic or $2 \% \mathrm{O}_{2}$ hypoxic conditions, $24 \mathrm{~h}$ following transfection. Subsequently, EdU staining (cells were incubated with the EdU reagent $\mathrm{A}$ for $2 \mathrm{~h}$ in the incubator at $37^{\circ} \mathrm{C}$, and subsequent procedures were performed according to the manufacturer's instructions; Cell-Light ${ }^{\mathrm{TM}}$ EdU Apollo567 In Vitro kit; Guangzhou RiboBio, Co., Ltd.) or cell viability (cells were incubated with the Cell Counting Kit (CCK)-8 reagent for 30 mins and OD was measured every 15 mins) (Cell Counting Kit- 8 assay; cat. no. HY-K0301; MedChem Express) was performed. The detailed procedures were conducted according to the manufacturers' instructions. Images were captured at x 200 magnification.
Colony formation assay. The cells were cultured with siXBP1s or siNC transfection (A549 cells), or XBP1s overexpression vector or control vector transfection (A549 and HBE cells) for $24 \mathrm{~h}$, then 300 cells/well were seeded into 6-well plates. After $\sim 2$ weeks, colony formation was assessed, and the cells were fixed with $4 \%$ paraformaldehyde for $15 \mathrm{mins}$, then stained with crystal violet for $15 \mathrm{mins}$, both at room temperature. Then, images of the colonies (>50 cells) were captured and manually calculated.

Transwell assay. Migration ability was evaluated using Transwell chambers with $8-\mu \mathrm{m}$ pores (Corning, Inc.). siXBP1s or siNC was transfected into the A549 cell line $\left(2.0 \times 10^{5}\right.$ cells/well) or XBP1s overexpression vector or control vector was transfected into the

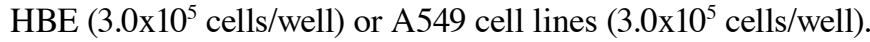
Subsequently, the cells were digested and a total of $1.0 \times 10^{4}$ cells suspended in $200 \mu \mathrm{l}$ culture medium (10\% FBS) were added to the upper chamber, and $600 \mu \mathrm{l}$ culture medium was added to the lower chamber, replacing the cell culture medium with fresh culture medium $24 \mathrm{~h}$ after suspension. The cells were cultured at $37^{\circ} \mathrm{C}$ in the incubator for $24 \mathrm{~h}$ with $21 \% \mathrm{O}_{2}$ (normoxic conditions) in the A549 and HBE cell lines, or $36 \mathrm{~h}$ with $21 \% \mathrm{O}_{2}$ or $2 \% \mathrm{O}_{2}$ (hypoxic conditions) in rescue experiments with the A549 cell line, until they were harvested. Then, the cells were fixed with $4 \%$ paraformaldehyde for 15 mins, then stained with crystal violet for $15 \mathrm{mins}$, both at room temperature. The cells were washed with PBS, then images of the migrated cells were captured (TE2000 U; light; Nikon Corporation) at x200 magnification.

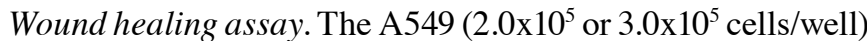
or HBE cell lines $\left(3.0 \times 10^{5}\right.$ cells/well) were seeded in a 6 -well plate and transfected $24 \mathrm{~h}$ later with siXBP1s or siNC, or XBP1s overexpression or control vector, respectively. When the cells reached $80-90 \%$ confluence, a wound was generated using a pipette tip. Then, the cells were cultured for $24 \mathrm{~h}$ with serum-free medium. The images of the cells were captured at 0 and $24 \mathrm{~h}$, at x200 magnification (TE2000 U; Nikon Corporation). The widths of the wound were recorded, and wound closure was calculated as [wound width (0-24 h)/wound width (0 h)] x100\%.

Flow cytometry. The A549 $\left(2.0 \times 10^{5}\right.$ or $3.0 \times 10^{5}$ cells/well $)$ or HBE cell lines $\left(3.0 \times 10^{5}\right.$ cells/well $)$ were seeded in a 6 -well plate and transfected $24 \mathrm{~h}$ later with siXBP1s or siNC, or XBP1s overexpression or control vector, respectively. The cells were cultured for $24 \mathrm{~h}$ with $21 \% \mathrm{O}_{2}$ (normoxic conditions) in the A549 and $\mathrm{HBE}$ cell lines, or for $36 \mathrm{~h}$ with $21 \% \mathrm{O}_{2}$ or $2 \% \mathrm{O}_{2}$ (hypoxic conditions) in rescue experiments with the A549 cell line. Then, the cells were collected, washed three times with cold PBS and suspended in $300 \mu \mathrm{l}$ binding buffer. Next, the cells were stained with $3 \mu \mathrm{l}$ Annexin V-FITC and $3 \mu \mathrm{l}$ PI at room temperature for 30 mins with an apoptosis detection kit (cat. no. 556547; BD Pharmingen; BD Biosciences).

Statistical analysis. All the quantitative data are presented as the mean \pm SD. GraphPad Prism (V8.0) was used for statistical analysis. Statistical significance was determined using an unpaired Student's t-test for comparisons between two groups, while one-way ANOVA followed by Bonferroni post hoc 
A

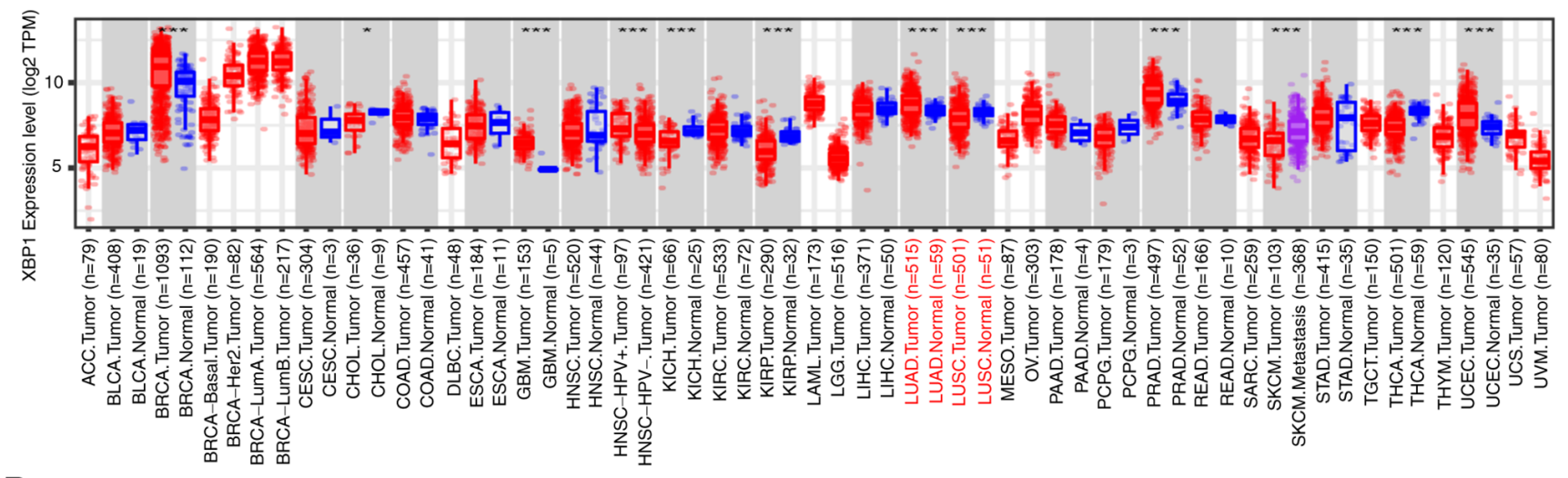

B
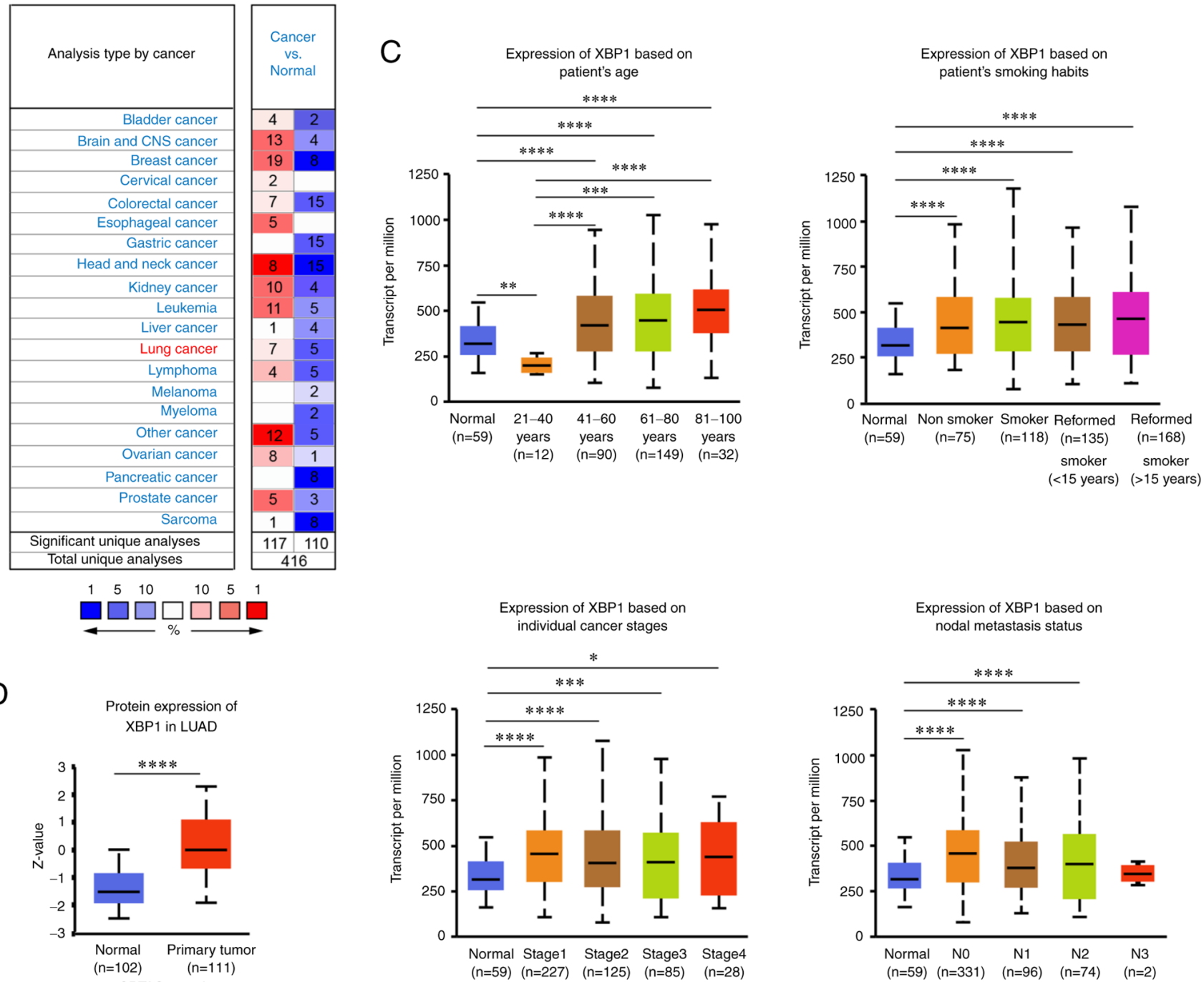

Figure 1. Expression profile of XBP1 in various types of cancer and the association between XBP1 and clinical characteristics in patients with LUAD. (A) The mRNA expression level of XBP1 in different types of cancer from The Cancer Genome Atlas using the TIMER2.0 database. The red color represents cancer tissue and the blue color represents normal tissue. (B) The expression level of XBP1 in various types of cancer compared with that in normal tissues using the ONCOMINE database. (C) Expression level of XBP1 in patients with different clinical features using the UALCAN database. (D) Protein expression level of XBP1 in patients with LUAD from the CPTAC using the UALCAN database. ${ }^{*} \mathrm{P}<0.05,{ }^{* *} \mathrm{P}<0.01,{ }^{* * * *} \mathrm{P}<0.001,{ }^{* * * * *} \mathrm{P}<0.001$. XBP1, X-box binding protein 1 ; LUAD, lung adenocarcinoma; CPTAC, Clinical Proteomic Tumor Analysis Consortium.

test was used for comparisons among more than two groups. All the experiments were performed independently at least three times. $\mathrm{P}<0.05$ was considered to indicate a statistically significant difference.

\section{Results}

Expression of XBP1s is increased in LUAD and A549 cell line. Analysis of data from the TIMER2.0, ONCOMINE and 
A

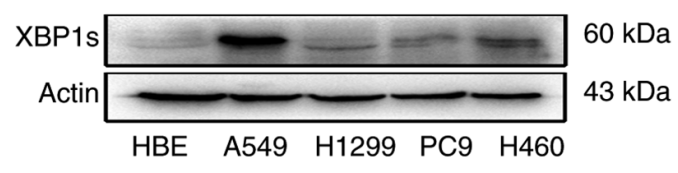

B

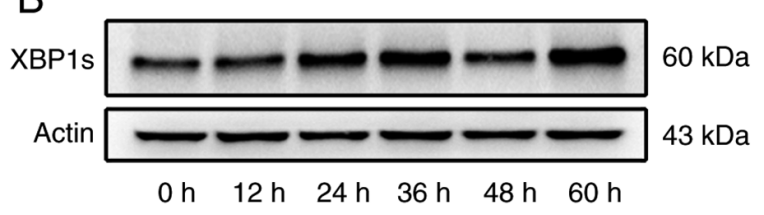

C

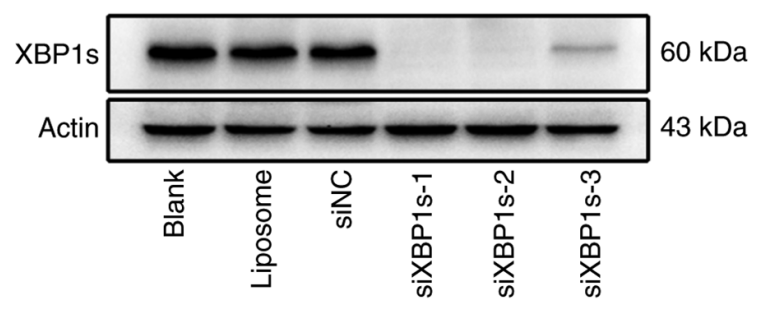

D

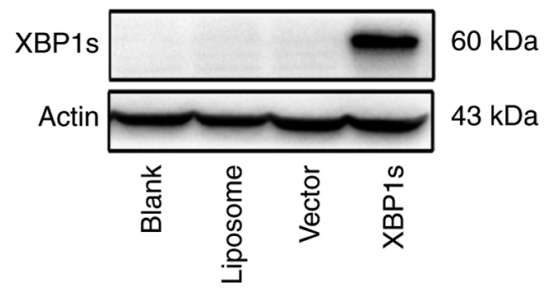

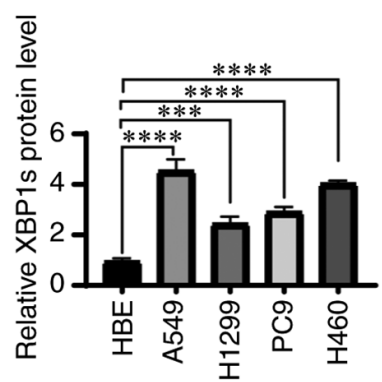
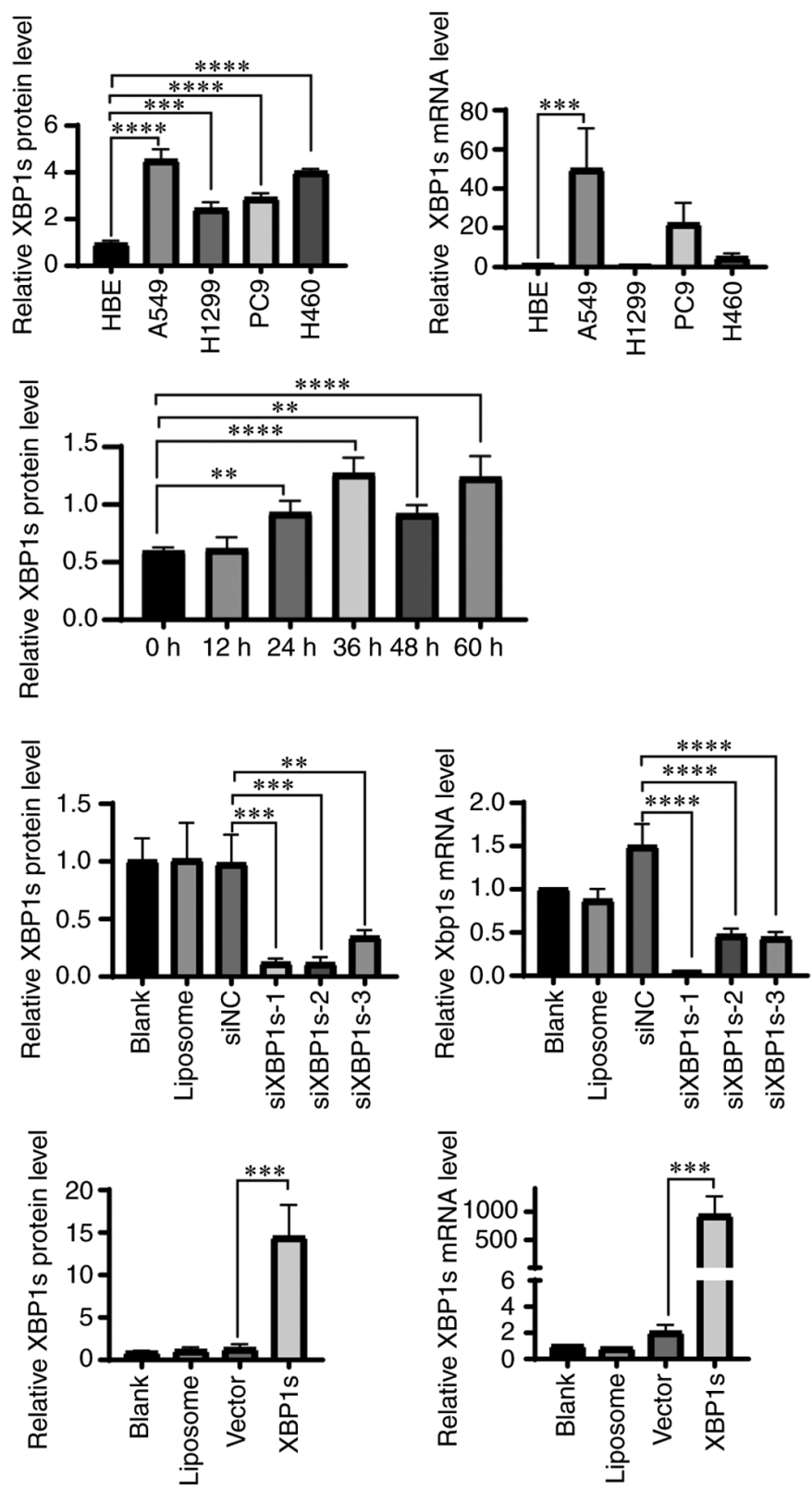

Figure 2. High expression level of XBP1s in the A549 cell line. (A) XBP1s protein and mRNA expression level was analyzed in the normal HBE and the A549, H1299, PC9 and H460 lung cancer cell lines. The protein expression level was subsequently analyzed using densitometry. (B) XBP1s protein expression level was measured using western blot analysis in the A549 cell line cultured under hypoxic conditions for the indicated times, and subsequently analyzed using densitometry. (C) Protein and mRNA expression levels of XBP1s was measured in the A549 cell line following no transfection (blank), transfection with liposome, siNC and siXBP1s-1/2/3. (D) Protein and mRNA expression levels of XBP1s was measured in the HBE cell line following no transfection (blank), transfection with liposome, control vector or XBP1 overexpression vector. $\mathrm{n}=3$. The data are presented as the mean $\pm \mathrm{SD}$. ${ }^{* * *} \mathrm{P}<0.01,{ }^{* * *} \mathrm{P}<0.001,{ }^{* * * *} \mathrm{P}<0.0001$. XBP1s, spliced X-box binding protein 1; LUAD, lung adenocarcinoma; NC, negative control; si, small interfering.

UALCAN databases revealed that XBP1 mRNA and protein expression level was highly expressed in various types of cancer compared with that in normal tissues, including LUAD (Fig. 1A, B and D, respectively). The mRNA expression level of XBP1 was significantly different in patients with different clinical features, including age, smoking habit, individual cancer stage and nodal metastasis status (Fig. 1C). Since XBP1s is a transcription factor, that is more active than XBP1u (28), the mRNA and protein expression level of XBP1s in the normal HBE, the A549, H1299 and PC9 LUAD, and the H460 large cell lung cancer cell lines was analyzed. XBP1s protein expression level was significantly elevated in the A549, H1299, PC9 and H460 cell lines compared with that in the HBE cell line. In addition, XBP1s mRNA expression level was significantly elevated in the A549 cell line compared with that in the HBE cell line (Fig. 2A). Since tumor cells survive in a hypoxic microenvironment (29), the A549 cell line was cultured under $2 \% \mathrm{O}_{2}$ hypoxia to further investigate the expression level of XBP1s. The protein expression level of XBP1s in the A549 cell line was increased under hypoxic conditions. The protein expression level reached its peak at $36 \mathrm{~h}$, then declined, followed by another elevation at $60 \mathrm{~h}$ (Fig. 2B). To further investigate the role of XBP1s in LUAD, siRNA was designed to knockdown the expression level of XBP1s in the A549 cell line, which had the highest protein and mRNA expression level compared with that in 

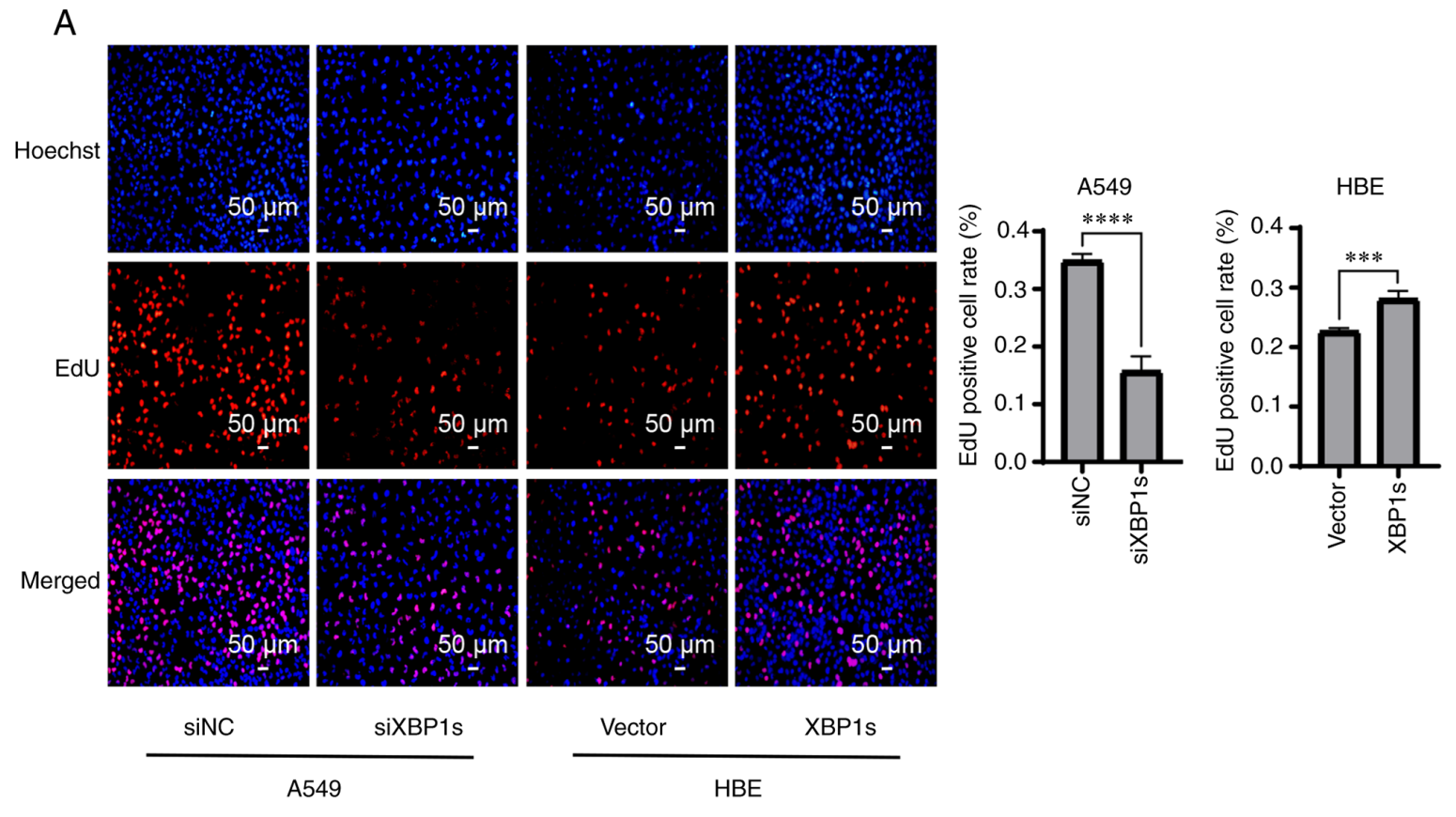

B
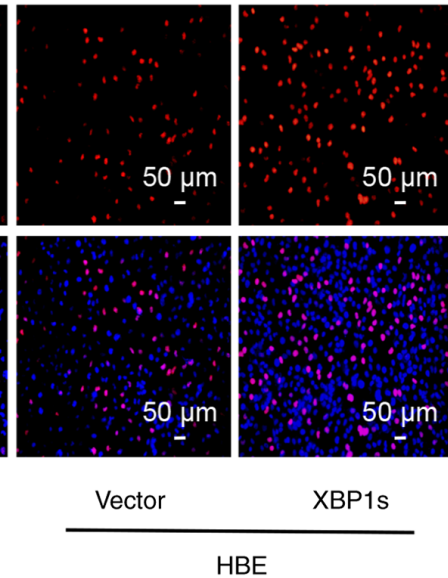

A549

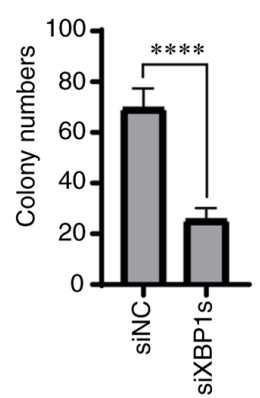

HBE
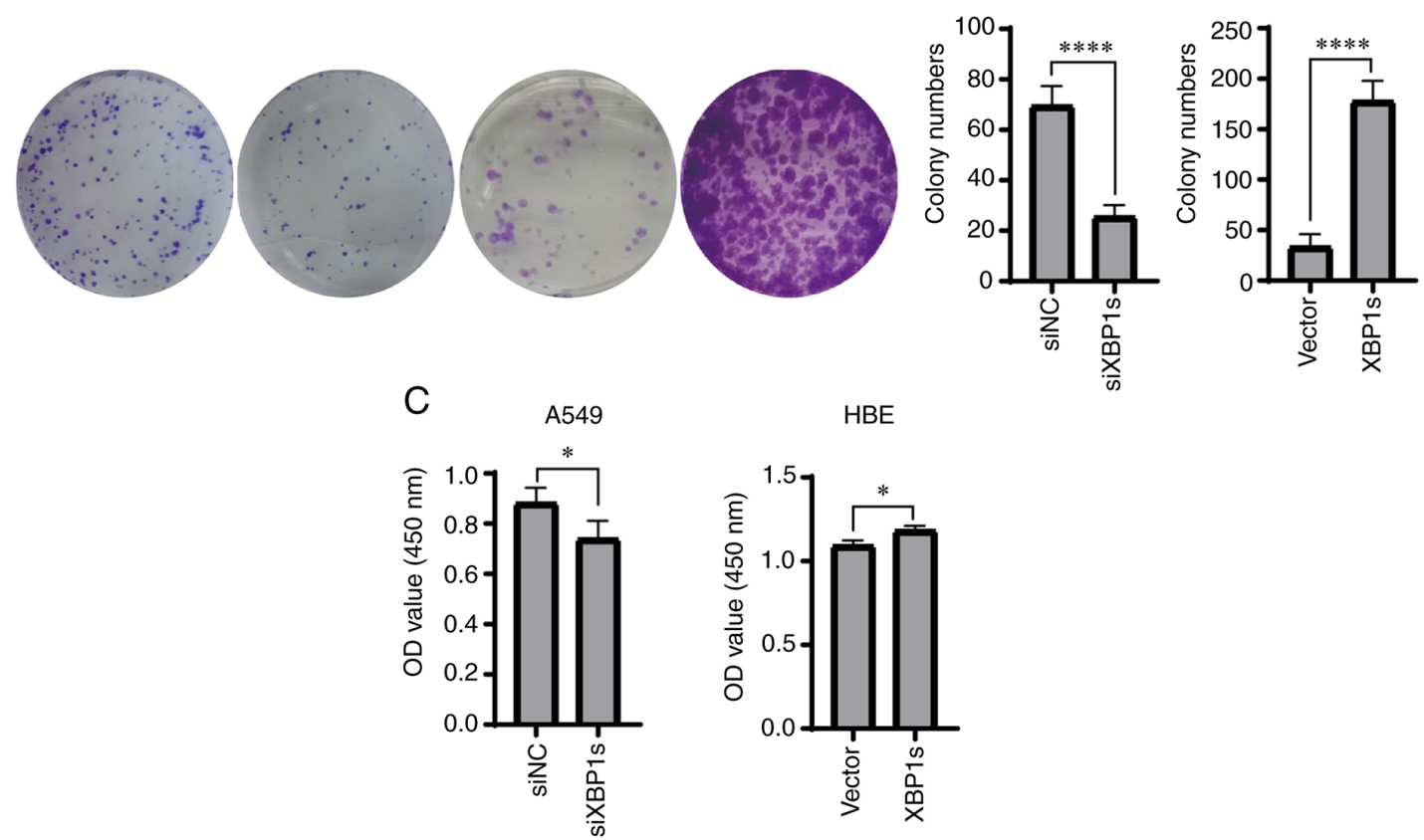

Figure 3. Knockdown of XBP1s expression in the A549 cell line decreases proliferation, colony formation and cell viability, while overexpressing XBP1s in the HBE cell line increases proliferation, colony formation and cell viability. The A549 cell line was transfected with siNC or siXBP1s, while the HBE cell line was transfected with control or XBP1s overexpression vector. Then, (A) EdU staining, (B) colony formation assay and (C) cytotoxicity assay was performed to determine the number of EdU-positive cells, number of colonies and cell viability, respectively. $n=3$. The data are presented as the mean \pm SD. ${ }^{*} P<0.05$, ${ }^{* * * *} \mathrm{P}<0.001,{ }^{* * * *} \mathrm{P}<0.0001$. XBP1s, spliced X-box binding protein 1; NC, negative control; si, small interfering; OD, optical density.

the HBE cell line. In addition, XBP1s overexpression vector was designed to overexpress XBP1s in the normal HBE cell line, which had the lowest mRNA and protein expression level. Western blot and RT-qPCR was performed to verify the knockdown and overexpression of XBP1s at the protein and mRNA level in the A549 (Fig. 2C) and HBE (Fig. 2D) cell lines, respectively. siXBP1s-1 significantly knocked down the expression level of XBP1s compared with that for siXBP1s-2 and -3 . These results indicated that the expression level of XBP1s was increased in LUAD and in the A549 cell line.
XBP1s is positively associated with proliferation, colony formation, and cell viability in both the A549 and HBE cell lines. The aforementioned results confirmed the high protein and mRNA expression level of XBP1s in the A549 cells; therefore, it was investigated whether XBP1s was associated with the proliferation, colony formation, and cell viability of the A549 and HBE cell lines. Proliferation was detected using EdU staining. The cells positive for EdU staining were stained red and were in a mitotic state. The proliferation rate of the A549 cells was significantly decreased when the expression level of XBP1s was knocked down. By contrast, it was 
A

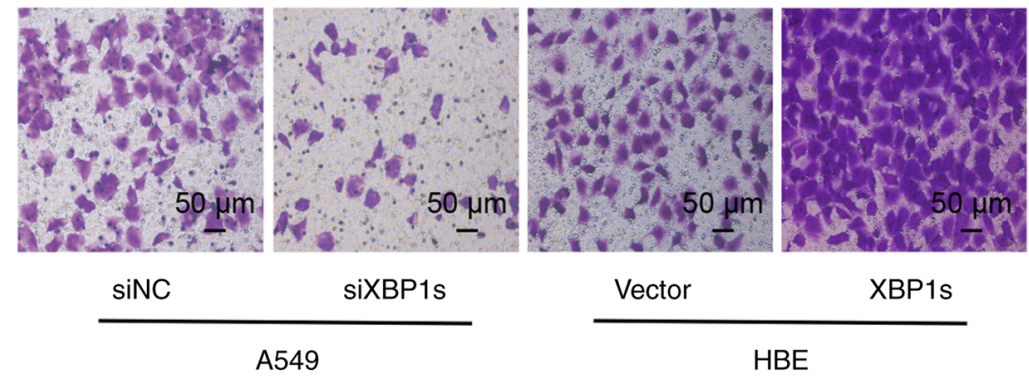

B
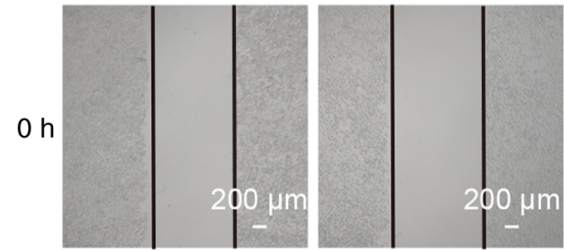

$24 \mathrm{~h}$
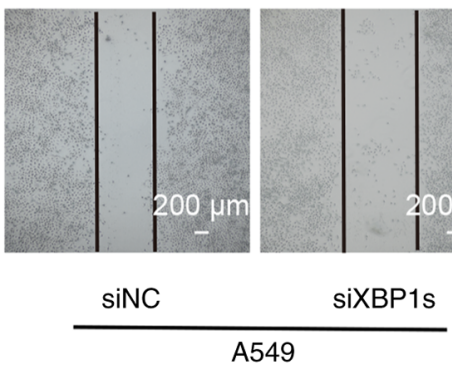

C
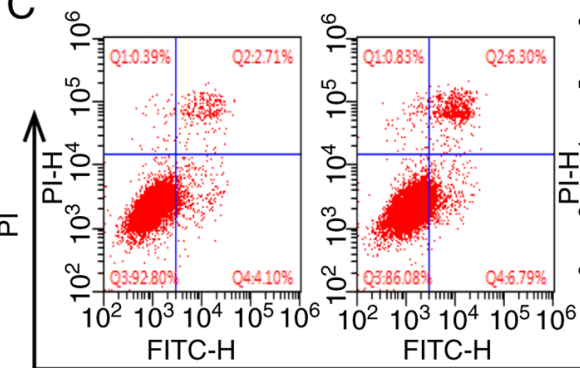

Annexin V-FITC

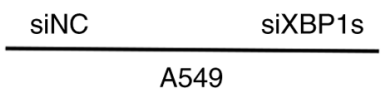

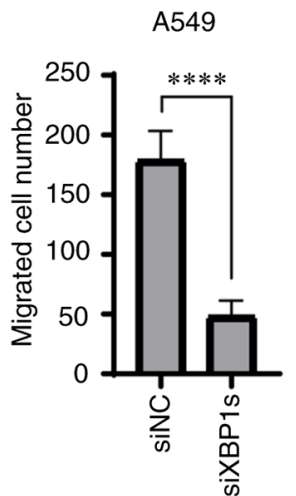

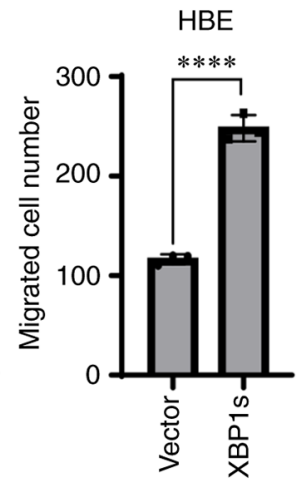

A549
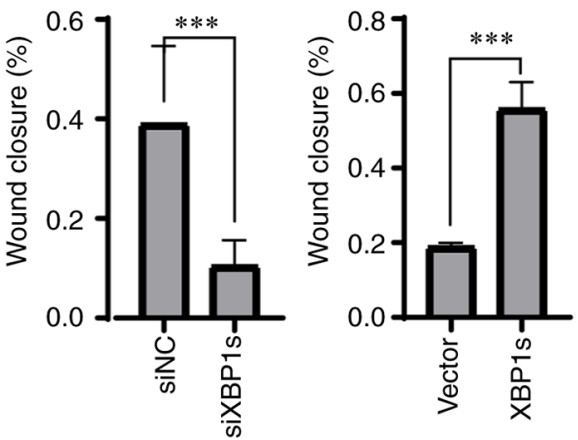

A549

HBE
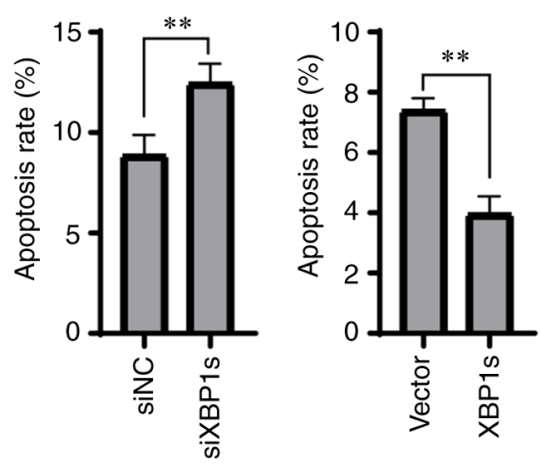

Figure 4. Knockdown of XBP1s expression in the A549 cell line decreases migration, wound healing rate, and increases apoptosis, while overexpressing XBP1s in the HBE cell line led to the opposite biological effects. The A549 cell line was transfected with siNC or siXBP1s, while the HBE cell line was transfected with control or XBP1s overexpression vector. Then, (A) Transwell assay, (B) wound healing assay and (C) flow cytometry was performed to analyze the migratory and wound healing ability, and apoptosis, respectively. The data are presented as the mean $\pm \mathrm{SD} . \mathrm{n}=3 .{ }^{* * *} \mathrm{P}<0.01,{ }^{* * * *} \mathrm{P}<0.001,{ }^{* * * *} \mathrm{P}<0.0001$. $\mathrm{XBP} 1 \mathrm{~s}$, spliced X-box binding protein 1; NC, negative control; si, small interfering.

significantly increased when XBP1s was overexpressed in the HBE cell line (Fig. 3A). Similarly, colony formation ability was significantly reduced in the A549 cell line following knock down of XBP1s expression, but was significantly increased in the HBE cells following overexpression of XBP1s (Fig. 3B). Similar results were also obtained when cell viability was measured (Fig. 3C). In conclusion, XBP1s was associated with proliferation, colony formation and cell viability in both the A549 and HBE cell lines.

$X B P 1 s$ is associated with migration, invasion and apoptosis in the A549 and HBE cell lines. In addition to proliferation, abnormally increased migration and invasion abilities and decreased apoptosis are also crucial factors that drive the worsening of tumor development $(30,31)$. Therefore, it was investigated whether XBP1s was associated with migration, invasion and apoptosis in the LUAD cell line, and in the normal HBE cell line as a control. Transwell assay demonstrated that knock down of XBP1s expression decreased the numbers of migrating A549 cells, while overexpressing XBP1s resulted in increased numbers of migrating HBE cells (Fig. 4A). A wound healing assay demonstrated that the wound healing ability of the cells was decreased following knock down of XBP1s expression in the A549 cell line and was increased following overexpression of XBP1s in the HBE cell line (Fig. 4B). Annexin-V FITC/PI double staining and flow cytometry was 
A

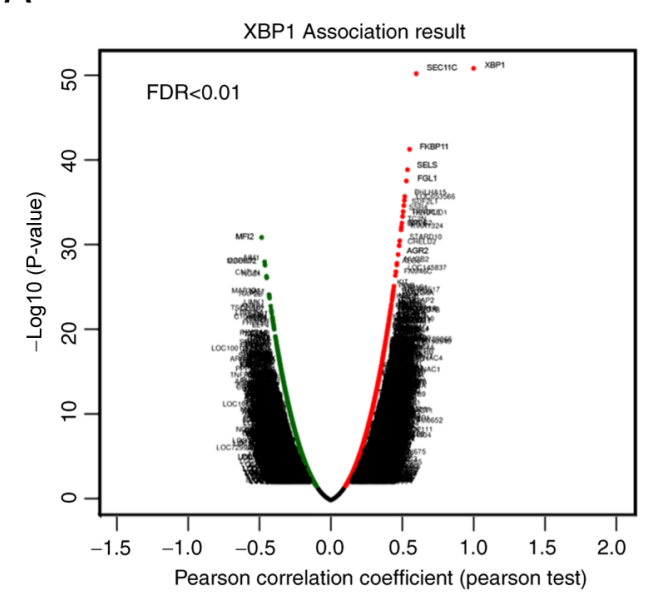

C

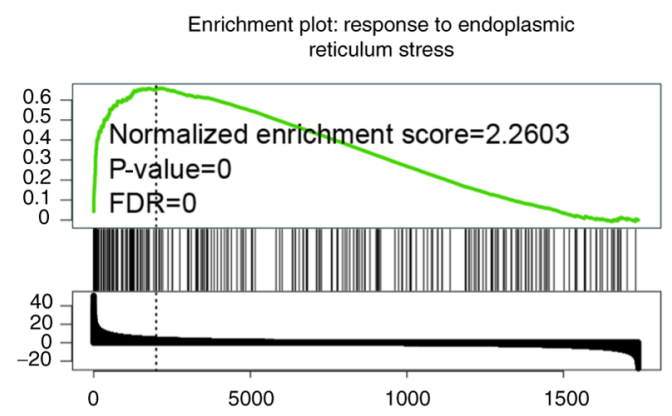

D

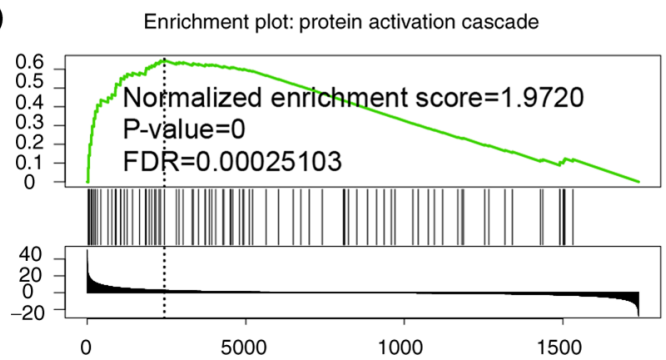

B

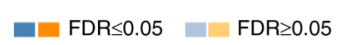

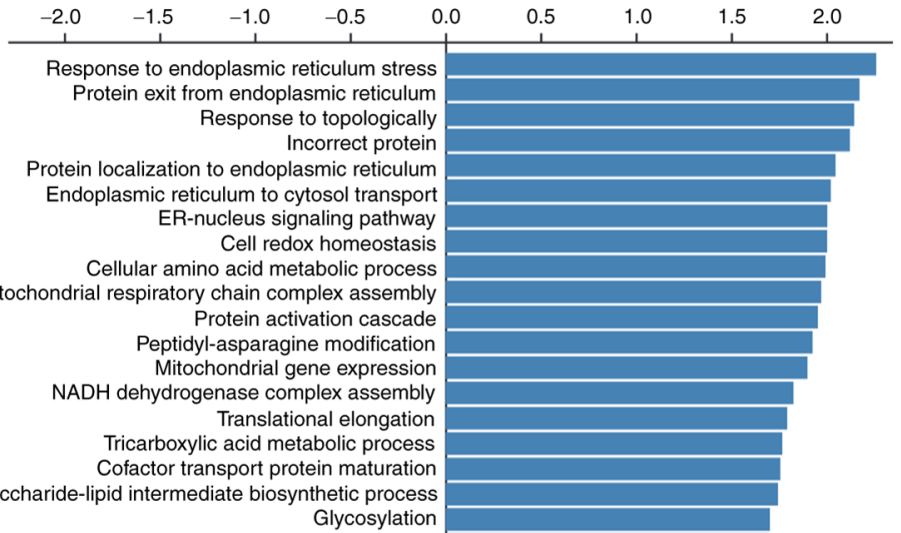

Cellular modified Antibiotic metabolic process Cellular modified amino acid metabolic process
Liposaccharide metabolic process

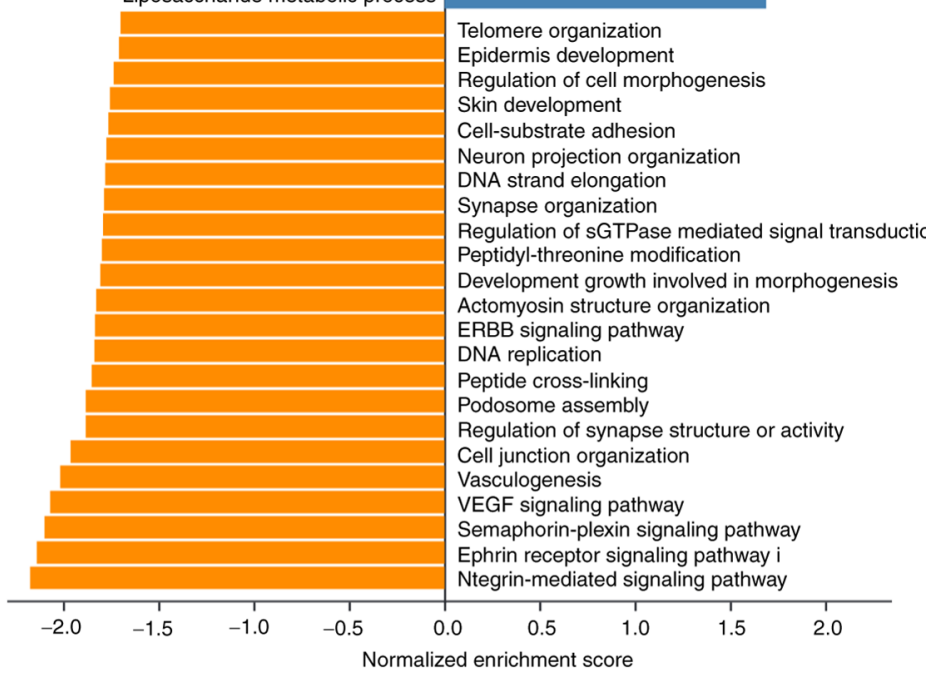

Telomere organization

Regulation of cell morphogenesis Skin development

Pyulation of sGTPase mediated signal transduction

Peptidyl-threonine modification

Development growth involved in morphogenesi ucture organization

Regulation of synapse structure or activity

Cell junction organization

Vasculogenesis

Normalized enrichment score

Figure 5. GO and pathway enrichment analysis of genes associated with XBP1. (A) Volcano plot representing genes highly associated with XBP1 using the LinkedOmics database. (B) GO (biological processes) analysis of genes associated with XBP1. GSEA pathway enrichment revealed the genes associated with XBP1 were enriched with response to (C) endoplasmic reticulum stress and (D) protein activation cascade. XBP1s, spliced X-box binding protein 1; GO, Gene Ontology; FDR, false discovery rate.

performed to detect apoptosis in the cell lines. The apoptosis rate was increased in the A549 cell line following knock down of XBP1s expression and decreased in the HBE cell line following overexpression of XBP1s (Fig. 4C). These results showed that XBP1s was associated with migration, invasion and apoptosis in the A549 and HBE cell lines.

$p$-JNK is the downstream target of XBPIs. To investigate the possible pathways that mediate the biological effects of XBP1s, GO and pathway enrichment analysis of the genes associated with XBP1 was analyzed using the LinkedOmics database. A total of 515 patients with LUAD from TCGA were analyzed. Genes that were associated with high XBP1 expression were found using the Volcano plot (Fig. 5A). The GO terms from the genes associated with XBP1, based on the LUAD samples revealed that these genes were most frequently involved in protein processing in the endoplasmic reticulum (Fig. 5B). GSEA pathway enrichment revealed that both endoplasmic reticulum stress (Fig. 5C) and protein activation cascade (Fig. 5D) were enriched with high expression of XBP1. As aforementioned, XBP1 is a transcription factor during ERS; therefore, it would be important to identify genes enriched in ERS. With respect to the enrichment of protein activation cascade, the MAPK pathway could be a possible candidate. In addition, activation of the MAPK pathway involves protein phosphorylation cascade and the MAPK pathway is a classic tumor-related pathway (32). Furthermore, when the pathways enriched in NSCLC were analyzed, the MAPK pathway was associated (Fig. S1A). To further identify whether the MAPK pathway was associated with XBP1, correlation analysis of XBP1 and the three main genes in the MAPK pathway was analyzed using a Pearson's correlation 
A

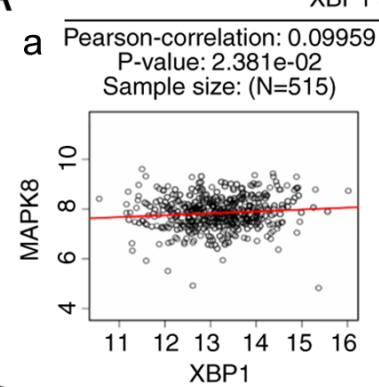

D

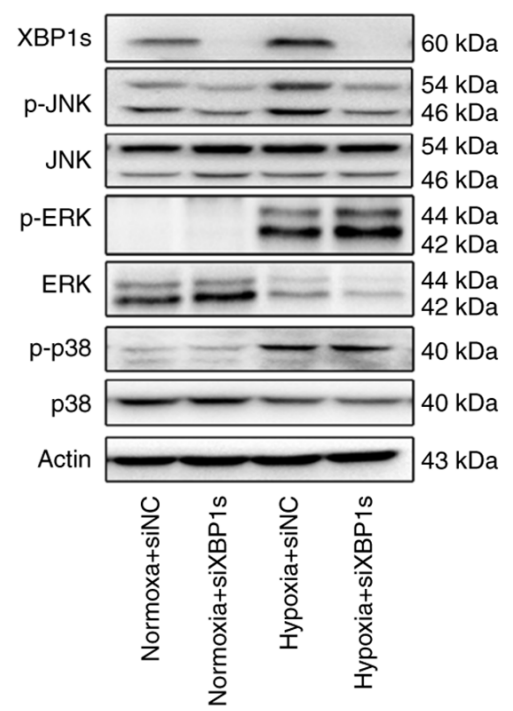

$\mathrm{B}$

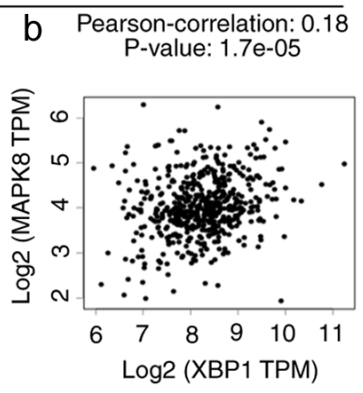

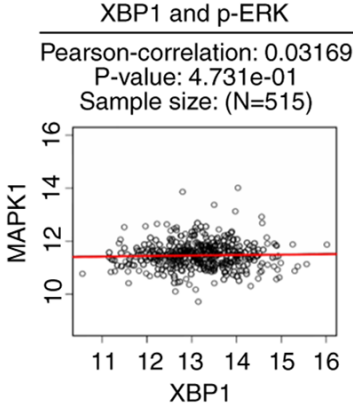

C

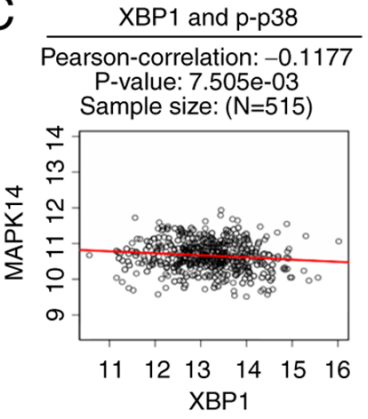

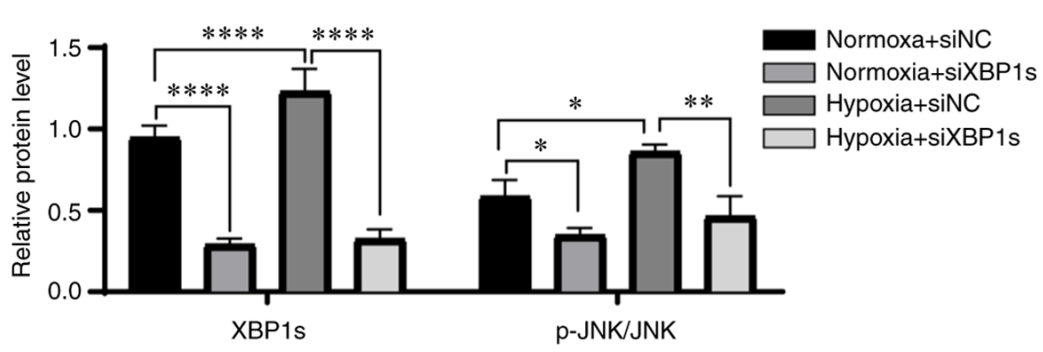

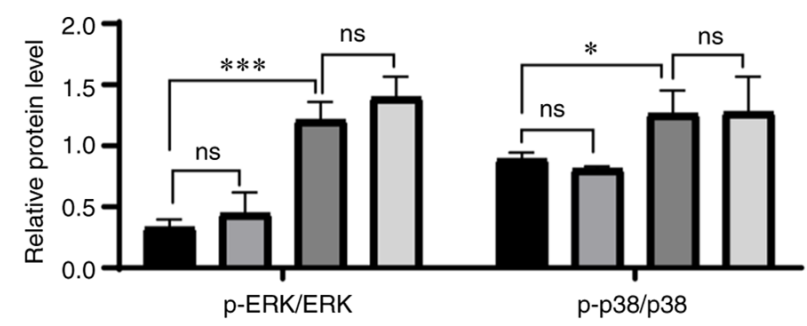

Figure 6. Verification of p-JNK MAPK as the downstream effector of XBP1s in the A549 cell line. Correlation analysis between XBP1 and p-JNK (MAPK8) using (A-a) LinkedOmics and (A-b) GEPIA databases. (B) Correlation analysis between XBP1 and p-ERK (MAPK1), and (C) p-p38 (MAPK14) using the LinkedOmics database. (D) Western blot analysis was used to determine the expression level of XBP1s and proteins involved in the MAPK signaling pathway in the A549 cell line following knockdown of XBP1s expression under normoxic and hypoxic conditions. The protein expression was subsequently analyzed using densitometry. $\mathrm{n}=3$. The data are presented as the mean $\pm \mathrm{SD}$. ${ }^{*} \mathrm{P}<0.05,{ }^{* *} \mathrm{P}<0.01,{ }^{* * * *} \mathrm{P}<0.001,{ }^{* * * *} \mathrm{P}<0.0001$. $\mathrm{XBP} 1 \mathrm{~s}$, spliced $\mathrm{X}$-box binding protein 1 ; $\mathrm{NC}$, negative control; si, small interfering; p, phosphorylated.

test. p-JNK (MAPK8) was found to be the only highly positively correlated gene both via LinkedOmics (Pearson's correlation, 0.09959; $\mathrm{P}=2.381 \times 10^{-2}$ ) (Fig. 6A-a) and GEPIA database (Pearson's correlation, 0.18; $\mathrm{P}=1.7 \times 10^{-5}$ ) (Fig. 6A-b). p-ERK (MAPK1) was not correlated with XBP1s using the LinkedOmics database (Fig. 6B). p-p38 (MAPK14) was not positively correlated with XBP1s using the LinkedOmics database (Fig. 6C). Correlation analysis between XBP1 and p-ERK or p-p38 was also performed using the GEPIA database (Fig. S1B), and neither were correlated. To further verify the association between XBP1s and the MAPK pathway, the protein expression level of proteins in the MAPK pathway was analyzed following knockdown of XBP1s expression. Since tumor cells often grow in a hypoxic microenvironment and it was aforementioned that XBP1s reached its peak expression level at $36 \mathrm{~h}$ when cultured in $2 \% \mathrm{O}_{2}$, the cells transfected with siNC or siXBP1s were cultured under normoxic $\left(21 \% \mathrm{O}_{2}, 36 \mathrm{~h}\right)$ or hypoxic $\left(2 \% \mathrm{O}_{2}, 36 \mathrm{~h}\right)$ conditions and the protein expression level of proteins in the MAPK pathway was analyzed. p-JNK expression was highly consistent with the expression level of XBP1s. The protein expression level of both p-JNK and XBP1s were increased under hypoxic conditions compared with that in normoxic conditions. When XBP1s expression was knocked down in cells cultured under normoxic or hypoxic conditions, p-JNK expression level was also decreased under both normoxic and hypoxic conditions. The expression levels of p-ERK and p-p38 were both increased under hypoxic conditions compared with that in normoxic conditions, but showed no change when XBP1s expression was knocked down under both normoxic and hypoxic conditions (Fig. 6D). Taken together, $\mathrm{p}$-JNK rather than p-ERK or p-p38 was found to be the downstream target of XBP1s.

Inhibition of p-JNK mitigates the biological effects caused by overexpression of XBPIs. Since p-JNK was hypothesized to be the downstream target of XBP1s, it was investigated whether inhibition of p-JNK could mitigate the effects caused by XBP1s overexpression. The A549 cells were cultured under normoxia, hypoxia, hypoxia with XBP1s overexpression, hypoxia with XBP1s overexpression and inhibition of p-JNK with SP600125. Hypoxia increased the proliferation rate (Fig. 7A and B), cell viability (Fig. 7C), colony formation ability (Fig. 7D and E), numbers of migrated cells (Fig. 8A), invasion ability (Fig. 8B), and decreased the apoptosis rate (Fig. 8C) of the A549 cells. XBP1s overexpression under hypoxia further enhanced these effects. However, cells treated with the p-JNK inhibitor exhibited a notable decrease in these effects (Figs. 7A-E and 8A-C). Taken together, the results indicated that inhibiting $\mathrm{p}-\mathrm{JNK}$ 
A

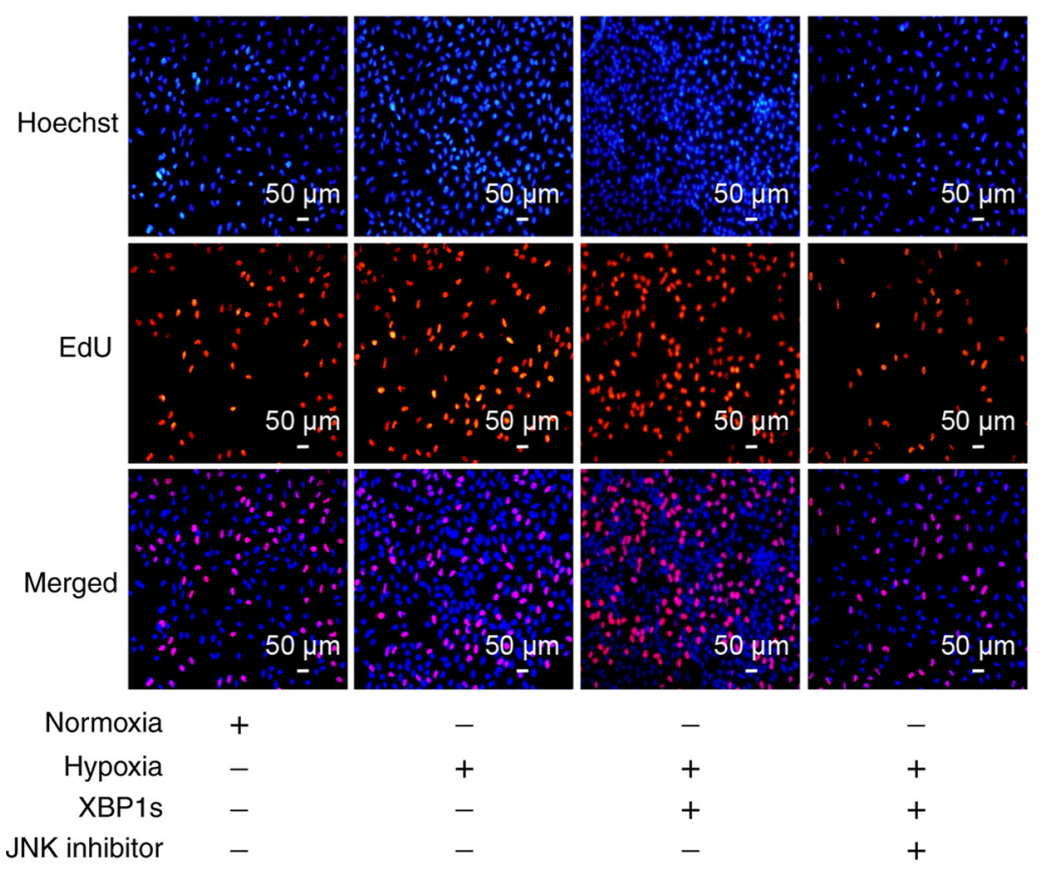

D

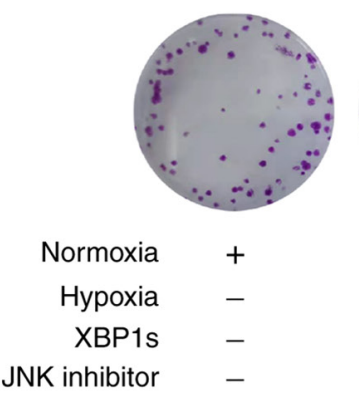

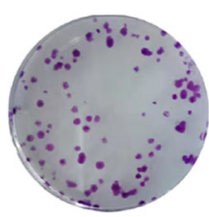

$-$

$+$

$-$

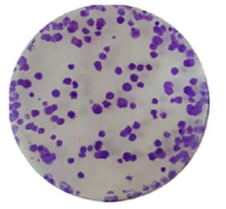

-
+
+
-

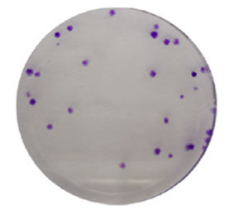

$-$

$+$

$+$
B

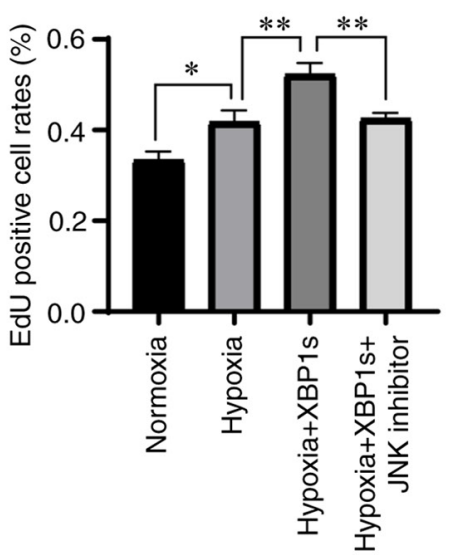

C

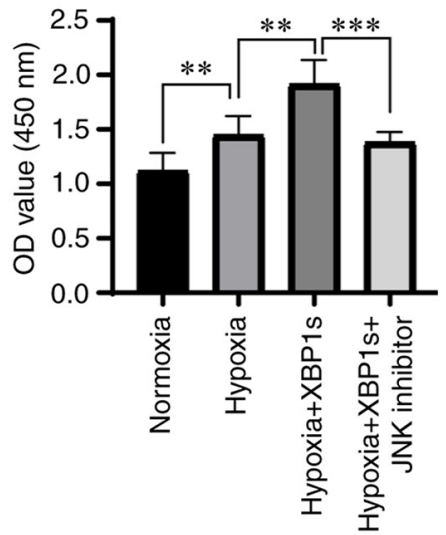

E

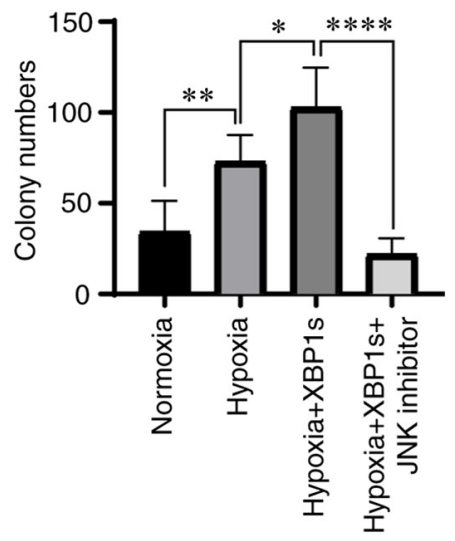

Figure 7. Inhibition of p-JNK mitigates the increased proliferation, cell viability and colony formation caused by overexpressing XBP1s. The A549 cell line was transfected with XBP1s overexpression or control vector, treated with or without JNK inhibitor and cultured under either normoxic or hypoxic conditions, then (A) EdU staining was performed and (B) EdU-positive cell rates were analyzed. (C) Cytotoxicity assay was performed to measure cell viability. (D) Colony formation assay were performed and (E) the number of colonies was analyzed. $\mathrm{n}=3$. The data are presented as the mean $\pm \mathrm{SD}$. ${ }^{*} \mathrm{P}<0.05$, ${ }^{* *} \mathrm{P}<0.01$, ${ }^{* * *} \mathrm{P}<0.001,{ }^{* * * *} \mathrm{P}<0.0001$. XBP1s, spliced $\mathrm{X}$-box binding protein 1; OD, optical density.

with SP600125 alleviated the biological effects of the overexpression of XBP1s.

\section{Discussion}

In brief, the results from the present study demonstrated that XBP1s promoted the development of LUAD via the p-JNK MAPK pathway. XBP1s was expressed at notably high levels in lung cancer tissues compared with that in normal tissues, and in the A549 cell line compared with that in the HBE,
H1299, PC9 and H460 cell lines. High expression of XBP1s promoted cell proliferation, colony formation, cell viability, migration, invasion, wound healing rate and reduction in apoptosis. Knockdown of XBP1s in the A549 cells resulted in the impairment of these prosurvival effects, while overexpression of XBP1s in the HBE cell line resulted in enhancement of these effects. Further investigation revealed that p-JNK was the downstream effector of XBP1s. Inhibition of p-JNK with SP600125 abolished the increased prosurvival effects caused by overexpressing XBP1s. 
A

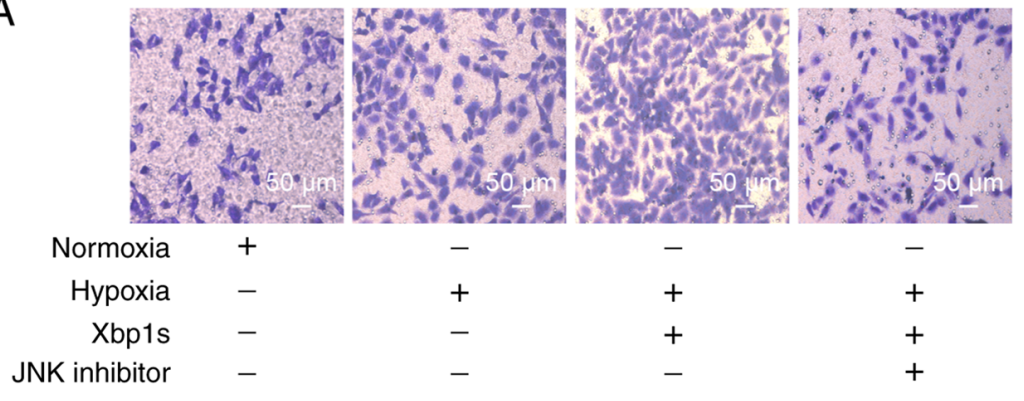

B
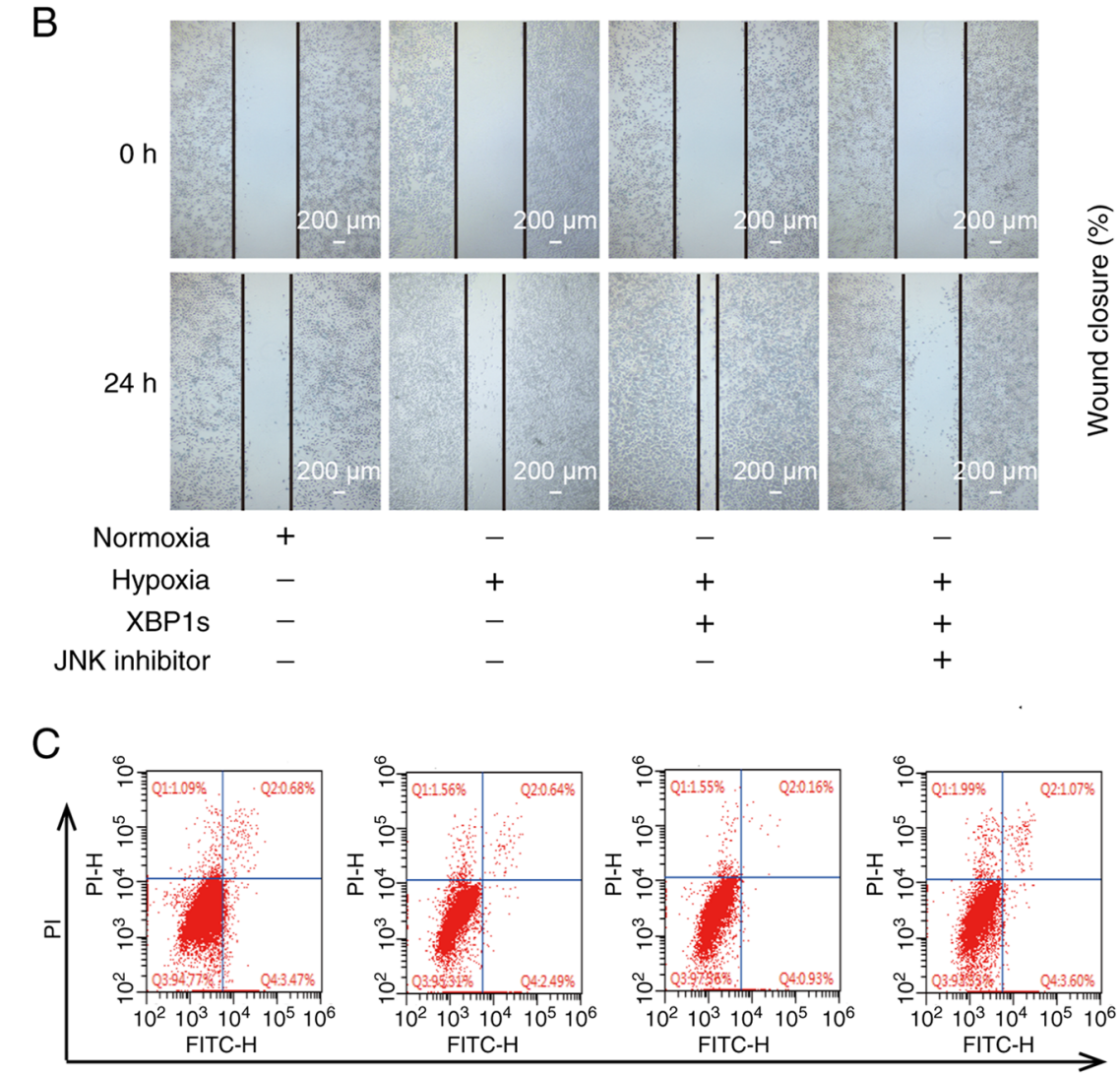

$\begin{array}{rr}\text { Normoxia } & + \\ \text { Hypoxia } & - \\ \text { XBP1s } & - \\ \text { JNK inhibitor } & -\end{array}$
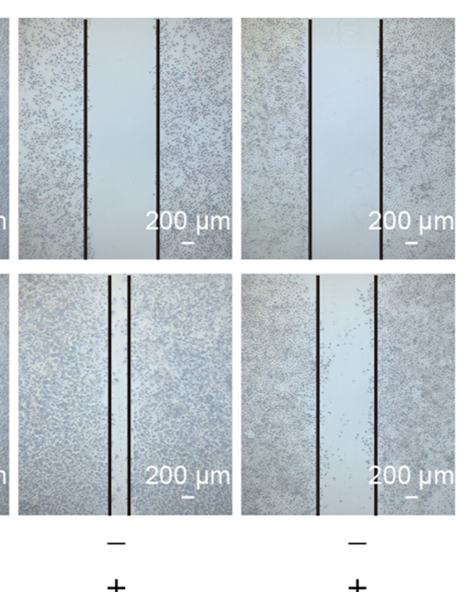

$+$

$+$

$-$
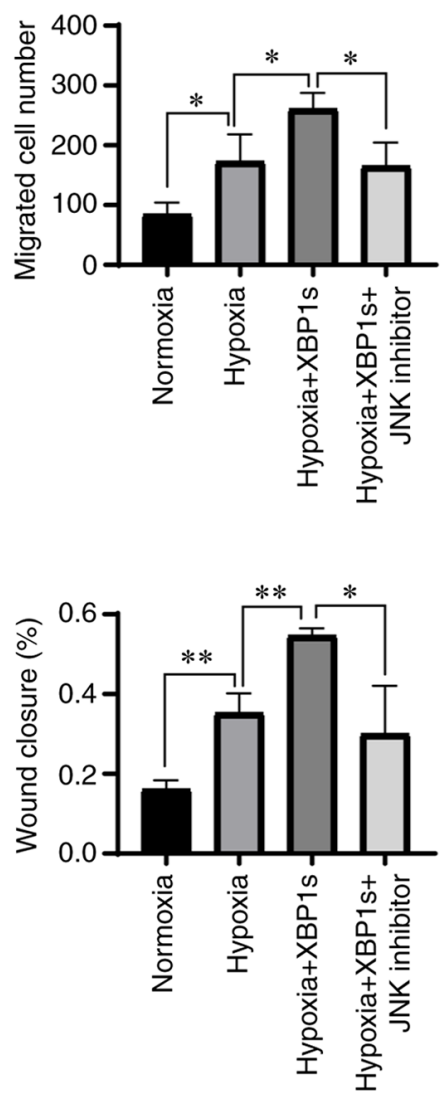

Figure 8. Inhibition of p-JNK mitigates the increased migration, wound healing rate, and the decreased apoptosis caused by overexpressing XBP1s. The A549 cell line was transfected with XBP1s overexpression or control vector, treated with or without JNK inhibitor and cultured under either normoxic or hypoxic conditions. Then (A) Transwell assay, (B) wound healing assay and (C) flow cytometry were performed and migration and apoptosis was measured and quantified. $\mathrm{n}=3$. The data are presented as the mean $\pm \mathrm{SD}$. ${ }^{*} \mathrm{P}<0.05,{ }^{* *} \mathrm{P}<0.01,{ }^{* * * * *} \mathrm{P}<0.0001$. $\mathrm{XBP} 1 \mathrm{~s}$, spliced $\mathrm{X}$-box binding protein 1 .

Targeting XBP1s for cancer therapy has been studied in numerous malignancies. In the tumor microenvironment, XBP1s cooperated with HIF1 $\alpha$ to promote cell survival and thus enabled some tumor subtypes to grow under hypoxic conditions (33). In triple-negative breast cancer, XBP1s was activated and drove tumorigenicity by forming a transcriptional complex with HIF1 $\alpha$ and regulating its expression (10). In ovarian cancer, XBP1 was highly expressed in T cells and tumor-associated dendritic cells, and upregulation of XBP1s expression impaired mitochondrial respiration and antitumor function $(9,34)$. In pancreatic ductal adenocarcinoma, XBP1s was associated with immune escape and metastasis (35). In prostate cancer, XBP1s expression was notably increased, promoted cancer development and was associated with prognosis $(8,36)$. In intestinal tumors, $\mathrm{XBP} 1$ determined the propensity of the epithelium to develop tumors by instructing a multilayered regenerative response in the intestinal epithelium (37). In glioblastoma multiform, IRE1-XBP1 was associated with tumor progression (38). The protein expression level of XBP1s is usually increased in these tumors and associated with tumorigenicity, metastasis and poor prognosis. In some malignancies, such as breast cancer, knockdown of XBP1s expression selectively blocked the growth of tumor cells (39). All these studies demonstrate that XBP1s promoted tumorigenesis, progression and prognosis. However, 
studies investigating how XBP1s functions in lung cancer are relatively limited. In the present study it was demonstrated that XBP1s was associated with the development of LUAD via the p-JNK MAPK pathway, providing further support for targeting XBP1s in LUAD treatment.

There are already drugs or inhibitors that target ERS for cancer therapy. Activation of ERS has been described in different human tumors, such as breast cancer, prostate cancer and kidney tumor, and in multiple cellular and animal models of cancer, such as murine cancer and prostate cancer cells (40). Tumor cells survive in a more hostile microenvironment, facing nutrient deprivation, oxygen limitation, high metabolic demand, and oxidative stress, and all these stimuli induce ERS in tumor cells. Sustained ERS endows malignant cells with greater tumorigenic, metastatic and drug-resistant ability (41). A large number of drugs or inhibitors that target IRE1 $\alpha$, PERK and ATF6 have been developed for cancer therapy. Among these IRE1 $\alpha$ inhibitors, STF-083010 and MKC-3946 are already at the preclinical stage. Other IRE1 $\alpha$ inhibitors, including MKC3946, B-I09 and MKC8866, were proven to induce apoptosis, and inhibit tumor growth $(42,43)$. These drugs and inhibitors provide exciting clinical perspectives for the treatment of various tumor subtypes, such as multiple myeloma xenografts and triple-negative breast cancer $(39,43,44)$. Based on the results from the current study, $\mathrm{XBP} 1 \mathrm{~s}$ played an important role in promoting survival in the A549 cell line. It is highly promising that IRE1 $\alpha$ inhibitors would be applicable for patients with LUAD.

The role of IRE1 $\alpha / \mathrm{XBP} 1 \mathrm{~s}$ in lung cancer has not been thoroughly studied; however, the role of MAPK in lung cancer has been investigated by numerous researchers, although the results are controversial. Some studies indicated that MAPK promoted the initiation and progression of tumors $(13,45)$, mediated proliferation and the antiapoptotic effects in $\operatorname{LUAD}(17,19)$, and was associated with poor survival and treatment resistance in lung cancer (46). However, other studies indicated that MAPK mediated migration inhibition (47) and the induction of cell apoptosis in tumors $(16,18,48)$. The results from the present study were consistent with most studies $(13,17,19,45,46)$. It was found that the MAPK pathway promoted tumor growth rather than suppressing it. In addition, it was found that p-JNK MAPK mediated the survival-promoting effects of XBP1s in the A549 cell line; thus, contributing to the development of LUAD.

There are also some limitations to the present study. It was found that $\mathrm{p}-\mathrm{JNK}$ was the downstream effector that mediated the pro-survival effects of XBP1s; however, whether it functioned via direct or indirect interaction requires further investigation. As a transcription factor, XBP1 is able to positively regulate protein phosphorylation (49), but how it promoted the phosphorylation of JNK remains unknown. The upstream activator of XBP1s, IRE1 was reported to be able to activate JNK during ERS (50). Whether there is an interplay between IRE1/JNK and XBP1s/p-JNK remains to be elucidated.

To conclude, it was found that XBP1s promoted the development of LUAD via the p-JNK MAPK pathway. Targeting $\mathrm{XBP} 1 \mathrm{~s} / \mathrm{p}-\mathrm{JNK}$ could be a potentially effective strategy for the treatment of LUAD.

\section{Acknowledgements}

Not applicable.

\section{Funding}

This study was funded by the National Natural Science Foundation of China (grant nos. 81973987, 81700051 and 81700052).

\section{Availability of data and materials}

The datasets used and/or analyzed during the current study are available from the corresponding author on reasonable request.

\section{Authors' contributions}

HJ was involved in conceptualization of the study, analysis, performed the experiments and writing the original draft of the manuscript. QJ was involved in the conceptualization of the study and methodology. YH and XLi were involved in the conceptualization of the study and revising and editing the manuscript. YX and XLiu contributed to the design of the study and critically reviewed the manuscript, revised and edited the manuscript. HJ and XLiu confirmed the authenticity of all the raw data. All authors read and approved the final manuscript.

\section{Ethics approval and consent to participate}

Not applicable.

\section{Patient consent for publication}

Not applicable.

\section{Competing interests}

The authors declare that they have no competing interests.

\section{References}

1. Bray F, Ferlay J, Soerjomataram I, Siegel RL, Torre LA and Jemal A: Global cancer statistics 2018: GLOBOCAN estimates of incidence and mortality worldwide for 36 cancers in 185 countries. CA Cancer J Clin 68: 394-424, 2018.

2. Devesa SS, Bray F, Vizcaino AP and Parkin DM: International lung cancer trends by histologic type: Male:Female differences diminishing and adenocarcinoma rates rising. Int J Cancer 117: 294-299, 2005 .

3. Chan BA and Hughes BG: Targeted therapy for non-small cell lung cancer: Current standards and the promise of the future. Transl Lung Cancer Res 4: 36-54, 2015.

4. Senft D and Ronai ZA: UPR, autophagy, and mitochondria crosstalk underlies the ER stress response. Trends Biochem Sci 40: 141-148, 2015.

5. Brenning G, Simonsson B, Kallander C and Ahre A: Pretreatment serum beta 2 -microglobulin in multiple myeloma. $\mathrm{Br}$ J Haematol 62: 85-93, 1986.

6. Shinya S, Kadokura H, Imagawa Y, Inoue M, Yanagitani K and Kohno K: Reconstitution and characterization of the unconventional splicing of XBPlu mRNA in vitro. Nucleic Acids Res 39: 5245-5254, 2011

7. Chen S, Chen J, Hua X, Sun Y, Cui R, Sha J and Zhu X: The emerging role of XBP1 in cancer. Biomed Pharmacother 127: 110069, 2020.

8. Sheng X, Nenseth HZ, Qu S, Kuzu OF, Frahnow T, Simon L, Greene S, Zeng Q, Fazli L, Rennie PS, et al: IRE1 $\alpha-X B P 1 s$ pathway promotes prostate cancer by activating c-MYC signaling. Nat Commun 10: 323, 2019.

9. Song M, Sandoval TA, Chae CS, Chopra S, Tan C, Rutkowski MR, Raundhal M, Chaurio RA, Payne KK, Konrad C, et al: IRE1 $\alpha$-XBP1 controls $T$ cell function in ovarian cancer by regulating mitochondrial activity. Nature 562: 423-428, 2018. 
10. Chen X, Iliopoulos D, Zhang Q, Tang Q, Greenblatt MB Hatziapostolou M, Lim E, Tam WL, Ni M, Chen Y, et al: XBP1 promotes triple-negative breast cancer by controlling the HIF1 $\alpha$ pathway. Nature 508: 103-107, 2014.

11. Luo Q, Shi W, Dou B, Wang J, Peng W, Liu X, Zhao D, Tang F, Wu Y, Li X, et al: XBP1-IGFBP3 signaling pathway promotes NSCLC invasion and metastasis. Front Oncol 11: 654995, 2021.

12. Kim EK and Choi EJ: Compromised MAPK signaling in human diseases: An update. Arch Toxicol 89: 867-882, 2015.

13. Cicchini M, Buza EL, Sagal KM, Gudiel AA, Durham AC and Feldser DM: Context-dependent effects of amplified MAPK signaling during lung adenocarcinoma initiation and progression. Cell Rep 18: 1958-1969, 2017.

14. Stutvoet TS, Kol A, de Vries EG, de Bruyn M, Fehrmann RS, Terwisscha van Scheltinga AG and de Jong S: MAPK pathway activity plays a key role in PD-L1 expression of lung adenocarcinoma cells. J Pathol 249: 52-64, 2019.

15. Cao X, Fang X, Malik WS, He Y, Li X, Xie M, Sun W, Xu Y and Liu X: TRB3 interacts with ERK and JNK and contributes to the proliferation, apoptosis, and migration of lung adenocarcinoma cells. J Cell Physiol 235: 538-547, 2020.

16. Shen H, Liu J, Wang Y, Lian H, Wang J, Xing L, Yan X, Wang J and Zhang X: Aflatoxin G1-induced oxidative stress causes DNA damage and triggers apoptosis through MAPK signaling pathway in A549 cells. Food Chem Toxicol 62: 661-669, 2013.

17. Du X, Wang S, Liu X, He T, Lin X, Wu S, Wang D, Li J, Huang W and Yang H: MiR-1307-5p targeting TRAF3 upregulates the MAPK/NF- $\kappa$ B pathway and promotes lung adenocarcinoma proliferation. Cancer Cell Int 20: 502, 2020.

18. Ong JY, Yong PV, Lim YM and Ho AS: 2-Methoxy1,4-naphthoquinone (MNQ) induces apoptosis of A549 lung adenocarcinoma cells via oxidation-triggered JNK and p38 MAPK signaling pathways. Life Sci 135: 158-164, 2015.

19. Hou XM, Zhang T, Da Z and Wu XA: CHPF promotes lung adenocarcinoma proliferation and anti-apoptosis via the MAPK pathway. Pathol Res Pract 215: 988-994, 2019.

20. Chen YY, Liu FC, Chou PY, Chien YC, Chang WS, Huang GJ, $\mathrm{Wu} \mathrm{CH}$ and Sheu MJ: Ethanol extracts of fruiting bodies of Antrodia cinnamomea suppress CL1-5 human lung adenocarcinoma cells migration by inhibiting matrix metalloproteinase-2/9 through ERK, JNK, p38, and PI3K/Akt signaling pathways. Evid Based Complement Alternat Med 2012: 378415, 2012

21. Rhodes DR, Kalyana-Sundaram S, Mahavisno V, Varambally R, Yu J, Briggs BB, Barrette TR, Anstet MJ, Kincead-Beal C, Kulkarni P, et al: Oncomine 3.0: Genes, pathways, and networks in a collection of 18,000 cancer gene expression profiles. Neoplasia 9: 166-180, 2007.

22. Li T, Fan J, Wang B, Traugh N, Chen Q, Liu JS, Li B and Liu XS TIMER: A web server for comprehensive analysis of tumor-infiltrating immune cells. Cancer Res 77: e108-e110, 2017

23. Chandrashekar DS, Bashel B, Balasubramanya SAH, Creighton CJ, Ponce-Rodriguez I, Chakravarthi BVSK and Varambally S: UALCAN: A portal for facilitating tumor subgroup gene expression and survival analyses. Neoplasia 19: 649-658, 2017

24. Vasaikar SV, Straub P, Wang J and Zhang B: LinkedOmics: Analyzing multi-omics data within and across 32 cancer types. Nucleic Acids Res 46: D956-D963, 2018.

25. Kanehisa M, Sato Y and Kawashima M: KEGG mapping tools for uncovering hidden features in biological data. Protein Sci 31: 47-53, 2022.

26. Tang Z, Li C, Kang B, Gao G, Li C and Zhang Z: GEPIA: A web server for cancer and normal gene expression profiling and interactive analyses. Nucleic Acids Res 45: W98-W102, 2017.

27. Livak KJ and Schmittgen TD: Analysis of relative gene expression data using real-time quantitative PCR and the 2(-Delta Delta C(T)) Method. Methods 25: 402-408, 2001

28. Yoshida $\mathrm{H}$, Matsui T, Yamamoto A, Okada T and Mori K: XBP1 mRNA is induced by ATF6 and spliced by IRE1 in response to ER stress to produce a highly active transcription factor. Cell 107 881-891, 2001.

29. Huang $\mathrm{CH}$, Chong KY and Lei KF: Analysis of the internal hypoxic environment in solid tumor tissue using a folding paper system. ACS Appl Mater Interfaces 13: 33885-33893, 2021

30. Jin X, Zhai B, Fang T, Guo X and Xu L: FXR1 is elevated in colorectal cancer and acts as an oncogene. Tumour Biol 37: 2683-2690, 2016.

31. Goldar S, Khaniani MS, Derakhshan SM and Baradaran B: Molecular mechanisms of apoptosis and roles in cancer development and treatment. Asian Pac J Cancer Prev 16: 2129-2144, 2015.
32. Drosten $\mathrm{M}$ and Barbacid M: Targeting the MAPK pathway in KRAS-Driven tumors. Cancer Cell 37: 543-550, 2020.

33. Xia Z, Wu S, Wei X, Liao Y, Yi P, Liu Y, Liu J and Liu J: Hypoxic ER stress suppresses $\beta$-catenin expression and promotes cooperation between the transcription factors XBP1 and HIF1 $\alpha$ for cell survival. J Biol Chem 294: 13811-13821, 2019.

34. Cubillos-Ruiz JR, Silberman PC, Rutkowski MR, Chopra S, Perales-Puchalt A, Song M, Zhang S, Bettigole SE, Gupta D, Holcomb K, et al: ER stress sensor XBP1 controls anti-tumor immunity by disrupting dendritic cell homeostasis. Cell 161: 1527-1538, 2015

35. Pommier A, Anaparthy N, Memos N, Kelley ZL, Gouronnec A, Yan R, Auffray C, Albrengues J, Egeblad M, Iacobuzio-Donahue CA, et al: Unresolved endoplasmic reticulum stress engenders immune-resistant, latent pancreatic cancer metastases. Science 360: eaao4908, 2018.

36. Sheng X, Arnoldussen YJ, Storm M, Tesikova M, Nenseth HZ, Zhao S, Fazli L, Rennie P, Risberg B, Wæhre H, et al: Divergent androgen regulation of unfolded protein response pathways drives prostate cancer. EMBO Mol Med 7: 788-801, 2015

37. Niederreiter L, Fritz TM, Adolph TE, Krismer AM, Offner FA, Tschurtschenthaler M, Flak MB, Hosomi S, Tomczak MF, Kaneider NC, et al: ER stress transcription factor Xbp1 suppresses intestinal tumorigenesis and directs intestinal stem cells. J Exp Med 210: 2041-2056, 2013.

38. Lhomond S, Avril T, Dejeans N, Voutetakis K, Doultsinos D, McMahon M, Pineau R, Obacz J, Papadodima O, Jouan F, et al: Dual IRE1 RNase functions dictate glioblastoma development. EMBO Mol Med 10: e7929, 2018.

39. Zhao N, Cao J, Xu L, Tang Q, Dobrolecki LE, Lv X, Talukdar M, Lu Y, Wang X, Hu DZ, et al: Pharmacological targeting of MYC-regulated IRE1/XBP1 pathway suppresses MYC-driven breast cancer. J Clin Invest 128: 1283-1299, 2018.

40. Clarke HJ, Chambers JE, Liniker E and Marciniak SJ: Endoplasmic reticulum stress in malignancy. Cancer Cell 25: 563-573, 2014.

41. Cubillos-Ruiz JR, Bettigole SE and Glimcher LH: Tumorigenic and immunosuppressive effects of endoplasmic reticulum stress in cancer. Cell 168: 692-706, 2017.

42. Wang M and Kaufman RJ: The impact of the endoplasmic reticulum protein-folding environment on cancer development. Nat Rev Cancer 14: 581-597, 2014.

43. Hetz C, Axten JM and Patterson JB: Pharmacological targeting of the unfolded protein response for disease intervention. Nat Chem Biol 15: 764-775, 2019.

44. Mimura N, Fulciniti M, Gorgun G, Tai YT, Cirstea D, Santo L, $\mathrm{Hu}$ Y, Fabre C, Minami J, Ohguchi H, et al: Blockade of XBP1 splicing by inhibition of IRElalpha is a promising therapeutic option in multiple myeloma. Blood 119: 5772-5781, 2012.

45. Liu P, Wang H, Liang Y, Hu A, Xing R, Jiang L, Yi L and Dong J: LINC00852 promotes lung adenocarcinoma spinal metastasis by targeting S100A9. J Cancer 9: 4139-4149, 2018.

46. Sato H, Schoenfeld AJ, Siau E, Lu YC, Tai H, Suzawa K, Kubota D, Lui AJW, Qeriqi B, Mattar M, et al: mapk pathway alterations correlate with poor survival and drive resistance to therapy in patients with lung cancers driven by ROS1 fusions. Clin Cancer Res 26: 2932-2945, 2020.

47. Zhou Q, Gui S, Zhou Q and Wang Y: Melatonin inhibits the migration of human lung adenocarcinoma A549 cell lines involving JNK/MAPK pathway. PLoS One 9: e101132, 2014

48. Liu T, Wu L, Wang D, Wang H, Chen J, Yang C, Bao J and Wu C: Role of reactive oxygen species-mediated MAPK and NF- $\kappa B$ activation in polygonatum cyrtonema lectin-induced apoptosis and autophagy in human lung adenocarcinoma A549 cells. J Biochem 160: 315-324, 2016.

49. QuickGO: Term GO:0001934. https://www.ebi.ac.uk/QuickGO/ term/GO:0001934. Accessed July 20, 2021.

50. Urano F, Wang X, Bertolotti A, Zhang Y, Chung P, Harding HP and Ron D: Coupling of stress in the ER to activation of JNK protein kinases by transmembrane protein kinase IRE1. Science 287: 664-666, 2000.

This work is licensed under a Creative Commons Attribution-NonCommercial-NoDerivatives 4.0 International (CC BY-NC-ND 4.0) License. 